\title{
Microscopic theory for long-range spatial correlations in lattice gas automata
}

\author{
H. J. Bussemaker* and M. H. Ernst \\ Institute for Theoretical Physics, University of Utrecht, P.O. Box 80006, 3508 TA, Utrecht, The Netherlands
}

(Received 21 February 1996)

\begin{abstract}
Lattice gas automata with collision rules that violate the conditions of semidetailed balance exhibit algebraic decay of equal-time spatial correlations between fluctuations of conserved densities. This is shown on the basis of a systematic microscopic theory. Analytical expressions for the dominant long-range behavior of correlation functions are derived using kinetic theory. We discuss a model of interacting random walkers with $x-y$ anisotropy whose pair correlation function decays as $1 / r^{2}$, and an isotropic fluid-type model with momentum correlations decaying as $1 / r^{2}$. The pair correlation function for an interacting random walker model with interactions satisfying all symmetries of the square lattice is shown to have $1 / r^{4}$ density correlations. Theoretical predictions for the amplitude of the algebraic tails are compared with the results of computer simulations. [S1063-651X(96)07006-7]
\end{abstract}

PACS number(s): 05.70.Ln, 05.40.+j, 05.20.Dd

\section{INTRODUCTION}

Closed, isolated physical systems, whose dynamics is described by a Hamiltonian $H(\Gamma)$, reach for long times a thermodynamic equilibrium state in which each microstate with total energy $E$ has equal weight $\rho(\Gamma) \sim \delta(H(\Gamma)-E)$ : the so-called microcanonical ensemble. When brought into contact with a heat reservoir, so that the energy is not fixed but fluctuates around an average value, the system is described by the canonical distribution $\rho(\Gamma) \sim e^{-\beta H(\Gamma)}$, with $\beta$ the inverse temperature. An essential observation is that in both cases the equilibrium distribution is completely known in terms of the Hamiltonian, without the need to explicitly solve the dynamics generated by $H(\Gamma)$.

The situation is quite different in the case of driven systems, where the dynamics does not satisfy the detailed balance condition, and prevents the system from reaching thermal equilibrium, e.g., due to an external driving field or due to heat reservoirs at different temperatures. An example of the latter is a fluid layer heated from above and cooled from below, so that a temperature gradient across the layer is maintained. After long times this system reaches a nonequilibrium steady state. The corresponding phase space distribution can only be determined by explicitly solving the dynamics, e.g., using kinetic theory [1].

It is helpful to study simple models for driven systems to gain insight into the nature of nonequilibrium steady states, and to compare theoretical predictions with the result of computer simulations. It is in fact simple to define models with stochastic dynamics that violate detailed balance.

A class of models that has been studied quite extensively in recent years is driven kinetic Ising models with Kawasakitype spin-flip dynamics [2] and certain particle hopping models [3]. For a recent review see Ref. [4]. Computer simulations have revealed algebraic decay of the density-density correlation function, i.e., $G(r) \simeq A / r^{n}$ for large $r$, in the stationary state. Although the exponent $n$ can be determined

\footnotetext{
*Permanent address: Institute for Physical Science and Technology, University of Maryland, College Park, MD 20742.
}

from symmetry considerations alone, using a Langevin equation approach $[2,5]$, there is currently no theory available that predicts the amplitude $A$ of the tail.

We propose lattice gas automata (LGA's) as an alternative class of simplified models that can be used to study the basic properties of nonequilibrium steady states. But more importantly, we present a systematic approximate theory for the large distance behavior of the correlation function of conserved quantities. Thus we are able to calculate the amplitude of the algebraic tails, starting from the microscopic definition of the model.

In addition to the type of lattice on which particles move and a required set of local conservation laws (particle density, momentum density, etc.), a LGA is defined by a set of stochastic transition probabilities that define the stochastic collision rules at each node. In the context of LGA's there is a distinction between collision rules that satisfy the condition of detailed or semidetailed balance [6] and rules that violate this condition. Semidetailed balance models reach for long times a completely factorized equilibrium state that is independent of the transition probabilities. However, to study nonequilibrium steady states of driven systems one needs to consider models with collision rules that violate semidetailed balance. Such collision rules are incompatible with a factorized state. Strong violation of semidetailed balance may even lead to spatial instability and pattern formation [7-9].

An advantage of LGA's over Ising-type models is that they can be used to model nonequilibrium states of fluids as well. In Ref. [10] it is explained how non-detailed-balance LGA fluids are to be considered as generalizations of driven diffusive systems.

Here we exclusively deal with LGA's having only stable modes, so that after long times a spatially homogeneous but correlated equilibrium state is reached. (Note that we use the term "equilibrium state" as a synonym for "steady state," to emphasize that we consider LGA's driven only through strictly local collision rules that are the same for each node and applied simultaneously to each node at each time step.) Such LGA's can be interpreted as effective models, whose dynamics represents a coarse-grained, mesoscopic description of a physical system kept out of thermal equilibrium. 
Due to their discrete nature LGA's are relatively easy to analyze, and studying their behavior will provide insight into the physics of nonequilibrium processes. On the other hand, many authors use LGA's lacking detailed balance to model physical phenomena, without analyzing how the lack of detailed balance may affect the validity of their conclusions. It is therefore important to have a fundamental understanding of the statistical mechanics of non-detailed balance LGA's.

To describe the correlations occurring in the correlated equilibrium state of non-detailed-balance LGA's a microscopic description beyond the Boltzmann equation is required. Bussemaker, Ernst, and Dufty [11] were the first to derive kinetic equations for LGA's at the level of pair correlations, by neglecting three-point and higher order correlation functions. This theory successfully predicts the magnitude of the pair correlations between occupation numbers at the same or at nearby nodes, as was shown in Ref. [11] by numerically evaluating the solution to the kinetic equations, and comparing it with simulation results.

Here we extend the analysis to large distances, and show that all LGA's lacking detailed balance possess spatial correlations between fluctuations of locally conserved quantities that decay algebraically for large distances. This is surprising since the collision rules only involve occupation numbers at the same node: zero-range interactions thus lead to infinite-range correlations. The mechanism that is responsible for the buildup of these long-range correlations involves the slow evolution of diffusive or hydrodynamic modes at large scales. It is the same mechanism that is responsible for the existence of the well-known long-time tails in hydrodynamic time correlation functions of equilibrium fluids, and the logarithmic density dependence of transport coefficients [12].

The organization of the paper is as follows. In Sec. II we recapitulate the kinetic equations of Ref. [11] in terms of excess correlation functions, and obtain an expression for the pair correlation function in terms of diffusive or hydrodynamic modes that resembles results derived from the phenomenological mode coupling theory. This expression is analyzed for interacting random walkers on a square lattice with $x-y$ anisotropy in Sec. III, and with the full square lattice symmetry in Sec. IV. In Sec. V we discuss a fluid-type LGA with full triangular lattice symmetry, which exhibits long-range momentum correlations. We end with a discussion in Sec. VI.

\section{RING KINETIC THEORY}

\section{A. Basic definitions}

We consider a LGA defined on a $d$-dimensional lattice of linear size $L$. The lattice has periodic boundary conditions and contains $V=L^{d}$ nodes. In this paper we will only use two-dimensional models with $d=2$. At each node $\mathbf{r}$ there are $b$ channels $\left(\mathbf{r}, \mathbf{c}_{i}\right)$ for moving particles with velocity $\mathbf{c}_{i}$ $(i=1, \ldots, b)$. We will consider two specific LGA's in this paper: (i) a model defined on a square lattice, where $\mathbf{c}_{i}$ are the nearest neighbor vectors $(\cos (i-1) \pi / 2, \sin (i-1) \pi / 2)$ with $i=1, \ldots, 4$, and (ii) a model defined on a triangular lattice, where $\mathbf{c}_{i}=(\cos (i-1) \pi / 3, \sin (i-1) \pi / 3)$ for $i=1, \ldots, 6$; in addition, there may be a channel $i=0$ for a rest particle with $\mathbf{c}_{0}=\mathbf{0}$. The absence (presence) of a particle in channel $\left(\mathbf{r}, \mathbf{c}_{i}\right)$ is denoted by Boolean occupation numbers $s_{i}(\mathbf{r})=\{0,1\}$.

The state of a node $\mathbf{r}$ is denoted by $s(\mathbf{r})=\left\{s_{i}(\mathbf{r})\right\}$. During the collision step of the LGA the precollision state $s(\mathbf{r})$ is replaced by a postcollision state $\sigma(\mathbf{r})$ at all nodes simultaneously, according to a stochastic process with transition probabilities $A_{s \sigma} \geqslant 0$. The $2^{b} \times 2^{b}$ matrix $A_{s \sigma}$ is normalized:

$$
\sum_{\sigma} A_{s \sigma}=1
$$

The collision step is followed by a propagation step during which a particle with postcollisional velocity $\mathbf{c}_{i}$ is moved from node $\mathbf{r}$ to a neighboring node $\mathbf{r}+\mathbf{c}_{i}$. The combined collision and propagation steps constitute a time evolution of the entire LGA from time $t$ to time $t+1$.

In most LGA's the collision rules satisfy certain local conservation laws. For instance, in a LGA describing the diffusive behavior of (interacting) random walkers, the number of particles at a node does not change during collision, but the distribution among velocity directions does. This conservation law is conveniently formulated in terms of the collisional invariant $a_{i}=1$. In a fluid-type LGA the local momentum at each node is also conserved during collision, and we have $a_{i}=\left\{1, \mathbf{c}_{i}\right\}$. Nonzero transition probabilities $A_{s \sigma}>0$ are only allowed if

$$
\sum_{i} a_{i} \sigma_{i}=\sum_{i} a_{i} s_{i}
$$

or, stated compactly, the matrix $A_{s \sigma}$ must satisfy

$$
\sum_{i} a_{i}\left(\sigma_{i}-s_{i}\right) A_{s \sigma}=0 .
$$

The transition matrix $A_{s \sigma}$ is said to satisfy the semidetailed-balance or Stueckelberg condition [13] if

$$
\sum_{s} A_{s \sigma}=1 .
$$

The stronger detailed-balance condition, $A_{s \sigma}=A_{\sigma s}$, implies semidetailed balance on account of the normalization (1). It can be shown that if Eq. (4) holds, the equilibrium distribution is completely factorized over all $b V$ channels $\left(\mathbf{r}, \mathbf{c}_{i}\right)$, and only depends on the microscopic state through global invariants, like the total number of particles or the total momentum [6]. Since the collision step does not change the value of these invariants, it follows that the equilibrium distribution is invariant under the collision step.

\section{B. Simple versus repeated ring approximation}

We restrict ourselves in this paper to properties of spatially homogeneous equilibrium states in LGA's lacking detailed balance. The quantities of interest are the average occupation number or single particle distribution function $f_{i}=\left\langle s_{i}(\mathbf{r})\right\rangle$ and the pair correlation function $\mathcal{G}_{i j}(\mathbf{r})$ $=\left\langle\delta s_{i}(\mathbf{r}) \delta s_{j}(\mathbf{0})\right\rangle$ with $\delta s_{i}(\mathbf{r})=s_{i}(\mathbf{r})-f_{i}$.

We give a short summary of necessary results derived in Ref. [11]. In semidetailed-balance models with zero-range 
interactions the average occupation number equals the Fermi distribution, and the pair correlation function has the diagonal form

$$
\mathcal{G}_{i j}(\mathbf{r})=\mathcal{G}_{i j}^{d}(\mathbf{r}) \equiv \delta_{i j} \delta(\mathbf{r}, \mathbf{0}) f_{i}\left(1-f_{i}\right)
$$

showing the absence of spatial or velocity correlations.

Next we consider models that violate semidetailed balance. It is convenient to introduce the excess pair correlation function

$$
\mathcal{C}_{i j}(\mathbf{r})=\mathcal{G}_{i j}(\mathbf{r})-\mathcal{G}_{i j}^{d}(\mathbf{r}),
$$

where a special role is played by the on-node correlations $\mathcal{C}_{i j} \equiv \mathcal{C}_{i j}(\mathbf{0})$. In the so-called simple ring approximation the average equilibrium occupations $\left\{f_{i}\right\}$ are the solution to the stationary nonlinear Boltzmann equation

$$
\Omega_{i}^{1,0}(f) \equiv \sum_{s \sigma}\left(\sigma_{i}-s_{i}\right) A_{s \sigma} F(s)=0
$$

where the nonlinear Boltzmann operator $\Omega_{i}^{1,0}(f)$ depends on the average occupations $f_{i}$ through the factorized distribution $F(s)$, defined as

$$
F(s)=\prod_{i} f_{i}^{s_{i}}\left(1-f_{i}\right)^{1-s_{i}}
$$

The source of all spatial correlations is the matrix $E$, which in the simple ring approximation is given by

$$
E_{i j}=\Omega_{i j}^{2,0} \equiv \sum_{s \sigma}\left(\delta \sigma_{i} \delta \sigma_{j}-\delta s_{i} \delta s_{j}\right) A_{s \sigma} F(s)
$$

Once $E_{i j}$ is known, the on-node correlations $\mathcal{C}_{i j}$ can be calculated from the stationary ring equation

$$
\mathcal{C}_{i j}=\sum_{i j k l} R_{i j, k \ell} E_{k l}
$$

The explicit form of the ring operator $R$, given in Ref. [11], is not needed here.

At a more sophisticated level, the repeated ring approximation, $\left\{f_{i}\right\}$ and $\left\{\mathcal{C}_{i j}\right\}$ are obtained as the solution to the stationary (generalized) Boltzmann equation

$$
\Omega^{1,0}(f)+\sum_{k<l} \Omega_{i, k l}^{1,2}(f) \mathcal{C}_{k l}=0
$$

where the term containing $\Omega_{i, k l}^{1,2}=\partial^{2} \Omega_{i}^{1,0} / \partial f_{k} \partial f_{l}$ describes corrections to $\Omega_{i}^{1,0}$. The on-node excess correlation function $\mathcal{C}$ couples the generalized Boltzmann equation (11) to the stationary ring kinetic equation (10), where the source matrix $E$ is now given by

$$
E_{i j}=\Omega_{i j}^{2,0}+\sum_{k<l} \Omega_{i j, k l}^{2,2} \mathcal{C}_{k l}+\sum_{k, l}(\mathbb{1}-\omega)_{i j, k l} \mathcal{C}_{k l},
$$

with $\Omega_{i j, k l}^{2,2}=\partial^{2} \Omega_{i j}^{2,0} / \partial f_{k} \partial f_{l}$ and $1_{i j, k l}=\delta_{i k} \delta_{j l}$. Furthermore, $\omega_{i j, k l}=(\mathbb{1}+\Omega)_{i k}(\mathbb{1}+\Omega)_{j l}$, where $\mathbb{1}$ is the unit matrix with $(1)_{i j}=\delta_{i j}$, and $\Omega$ is the linearized Boltzmann collision operator, defined as

$$
\Omega_{i j}=\frac{\partial \Omega_{i}^{1,0}}{\partial f_{j}}=\sum_{s \sigma}\left(\sigma_{i}-s_{i}\right) A_{s \sigma} F(s) \frac{\delta s_{j}}{f_{j}\left(1-f_{j}\right)} .
$$

For a derivation of these equations as well as a detailed discussion of how to obtain a (numerical) solution, we refer to Ref. [11]. Using the definition in Eq. (9) or (12) it can be shown that $E$ satisfies all local conservation laws of the model, i.e.,

$$
\langle a a \mid E\rangle \equiv\langle a|E| a\rangle \equiv \sum_{i j} a_{i} a_{j} E_{i j}=0
$$

We have found [11] that for models with local conservation laws the numerical difference between the simple and repeated ring values for $\mathcal{C}_{i j}$ is on the order of $10 \%$. Corrections to the Boltzmann value for $f_{i}$, as obtained from Eq. (11), are even smaller - typically $1 \%$.

As shown in Ref. [11] and the next subsection, all spatial correlations in the system are linear in the source term $E$. If this term vanishes, all correlations in the system vanish, and the equilibrium state is completely factorized. That the source term does indeed vanish if the collision rules satisfy the detailed-balance condition can be seen as follows. As noted above Eq. (5), $f_{i}$ is a Fermi distribution. Then the single node distribution (8) satisfies the relation $F(s) A_{s \sigma}=F(\sigma) A_{s \sigma}$. Using normalization (1) and semidetailed balance (4) it follows that $\Omega_{i j}^{2,0}=0$, and consequently $E_{i j}=0$, in both simple and repeated ring approximations. If the transition rates do not obey the semidetailed-balance condition (4) then $\Omega_{i j}^{2,0}$ is in general nonvanishing.

\section{Mode coupling formula}

Here we are concerned with correlation functions of conserved (hydrodynamic) densities. In diffusive models the only conserved density is the number density $\rho(\mathbf{r})$ $=\Sigma_{i} s_{i}(\mathbf{r})$. In fluid-type models the momentum density $\mathbf{g}(\mathbf{r})=\sum_{i} \mathbf{c}_{i} s_{i}(\mathbf{r})$ is conserved as well. We denote the conserved densities collectively as $a(\mathbf{r})=\sum_{i} a_{i} s_{i}(\mathbf{r})$. The hydrodynamic correlation functions are then expressed in terms of a scalar product:

$\mathcal{G}_{a}(\mathbf{r})=\langle\delta a(\mathbf{r}) \delta a(\mathbf{0})\rangle=\sum_{i j} a_{i} a_{j} \mathcal{G}_{i j}(\mathbf{r}) \equiv\langle a a \mid \mathcal{G}(\mathbf{r})\rangle$,

where the fluctuation $\delta a(\mathbf{r})=\sum_{i} a_{i} \delta s_{i}(\mathbf{r})$. The Fourier transform of the correlation function $\mathcal{G}_{i j}(\mathbf{r})$, defined by

$$
\hat{\mathcal{G}}_{i j}(\mathbf{q})=\sum_{\mathbf{r}} e^{-i \mathbf{q} \cdot \mathbf{r}} \mathcal{G}_{i j}(\mathbf{r})
$$

can be split as

$$
\hat{\mathcal{G}}_{i j}(\mathbf{q})=\delta_{i j} f_{i}\left(1-f_{i}\right)+\hat{\mathcal{C}}_{i j}(\mathbf{q})
$$

The constant contribution on the right hand side comes from the diagonal part defined in Eq. (5), and $\mathcal{C}_{i j}(\mathbf{q})$ denotes the Fourier transform of the excess correlation function defined in Eq. (6). In a similar manner, the susceptibility $\chi_{a}(\mathbf{q})$ is defined as the Fourier transform of $\mathcal{G}_{a}(\mathbf{r})$, i.e., 


$$
\chi_{a}(\mathbf{q})=\sum_{\mathbf{r}} e^{-i \mathbf{q} \cdot \mathbf{r}} \mathcal{G}_{a}(\mathbf{r})
$$

and split into two parts:

$$
\chi_{a}(\mathbf{q})=\chi_{a}^{d}+\Delta \chi_{a}(\mathbf{q}) .
$$

Its diagonal part $\chi_{a}^{d}$ is given by

$$
\chi_{a}^{d}=\sum_{i}\left(a_{i}\right)^{2} f_{i}\left(1-f_{i}\right)
$$

and the excess part $\Delta \chi_{a}(\mathbf{q})$ by

$$
\Delta \chi_{a}(\mathbf{q})=\sum_{i j} a_{i} a_{j} \hat{\mathcal{C}}_{i j}(\mathbf{q}) \equiv\langle a a \mid \hat{\mathcal{C}}(\mathbf{q})\rangle
$$

The main result of Ref. [10] describes the dominant behavior of the susceptibility at small wave number $(q \rightarrow 0)$ as

$$
\begin{aligned}
\Delta \chi_{a}(\mathbf{q})= & \sum_{\mu \nu}\left\langle a a \mid \widetilde{\psi}_{\mu}(\mathbf{q}) \widetilde{\psi}_{\nu}(-\mathbf{q})\right\rangle \frac{1}{1-e^{z_{\mu}(\mathbf{q})+z_{\nu}(-\mathbf{q})}} \\
& \times\left\langle\psi_{\mu}(\mathbf{q}) \psi_{\nu}(-\mathbf{q}) \mid E\right\rangle,
\end{aligned}
$$

which has the structure of a mode coupling formula. Here $\widetilde{\psi}_{\mu}(\mathbf{q})$ and $\psi_{\mu}(\mathbf{q})$ are the slow right and left (diffusive or hydrodynamic) eigenmodes of the LGA, determined by the eigenvectors of the lattice Boltzmann equation:

$$
\begin{gathered}
{\left[e^{z_{\mu}(\mathbf{q})+i \mathbf{q} \cdot \mathbf{c}}-1-\Omega\right] \widetilde{\psi}_{\mu}(\mathbf{q})=0,} \\
{\left[e^{z_{\mu}(\mathbf{q})+i \mathbf{q} \cdot \mathbf{c}}-1-\Omega^{T}\right] \psi_{\mu}(\mathbf{q})=0 .}
\end{gathered}
$$

Here $\Omega^{T}$ is the transpose of the linearized Boltzmann collision operator $\Omega$ in Eq. (13). The matrices $e^{i \mathbf{q} \cdot \mathbf{c}}$ and 1 are diagonal matrices with elements $\delta_{i j} e^{i \mathbf{q} \cdot \mathbf{c}_{i}}$ and $\delta_{i j}$, respectively. The eigenvalue or relaxation rate of the slow mode $\left\{\psi_{\mu}, \widetilde{\psi}_{\mu}\right\}$ is $z_{\mu}(\mathbf{q})$. For small $\mathbf{q}$ it behaves as $z_{\mu}(\mathbf{q}) \sim q^{2}$ for purely diffusive modes, and as $z_{\mu}(\mathbf{q}) \sim q$ for propagating sound modes. The right and left eigenmodes $\widetilde{\psi}_{\mu}(\mathbf{q})$ and $\psi_{\mu}(\mathbf{q})$ are $b$-dimensional vectors [or $(b+1)$-dimensional if the model admits states with a rest particle], with components $\widetilde{\psi}_{\mu i}$ and $\psi_{\mu i}$. They form a biorthonormal set, satisfying the orthogonality relation

$$
\left\langle\psi_{\mu}(\mathbf{q})\left|e^{i \mathbf{q} \cdot \mathbf{c}}\right| \widetilde{\psi}_{\nu}(\mathbf{q})\right\rangle \equiv \sum_{i} \psi_{\mu i}(\mathbf{q}) e^{i \mathbf{q} \cdot \mathbf{c}_{i}} \widetilde{\psi}_{\nu i}(\mathbf{q})=\delta_{\mu \nu}
$$

Note that all inner products in this article are defined without complex conjugation.

\section{Perturbation theory}

The small- $q$ behavior of the susceptibility $\chi_{a}(\mathbf{q})$ determines the long-range behavior of the corresponding correlation function $\mathcal{G}_{a}(\mathbf{r})$. We therefore write $\Delta \chi_{a}(\mathbf{q})$ as a Taylor expansion in powers of the wave number $q=|\mathbf{q}|$ :

$$
\Delta \chi_{a}(\mathbf{q})=\Delta \chi_{a}^{(0)}(\hat{\mathbf{q}})+q^{2} \Delta \chi_{a}^{(2)}(\hat{\mathbf{q}})+q^{4} \Delta \chi_{a}^{(4)}(\hat{\mathbf{q}})+\cdots .
$$

Explicit expressions for the functions $\Delta \chi_{a}^{(m)}(\hat{\mathbf{q}})$ occurring in Eq. (25) can be obtained by expanding the eigenvectors and eigenvalues in Eq. (23) in powers of $q$ :

$$
\begin{aligned}
& \psi_{\mu}(\mathbf{q})=\psi_{\mu}^{(0)}(\hat{\mathbf{q}})+(i q) \psi_{\mu}^{(1)}(\hat{\mathbf{q}})+(i q)^{2} \psi_{\mu}^{(2)}(\hat{\mathbf{q}})+\cdots, \\
& \widetilde{\psi}_{\mu}(\mathbf{q})=\widetilde{\psi}_{\mu}^{(0)}(\hat{\mathbf{q}})+(i q) \widetilde{\psi}_{\mu}^{(1)}(\hat{\mathbf{q}})+(i q)^{2} \widetilde{\psi}_{\mu}^{(2)}(\hat{\mathbf{q}})+\cdots, \\
& z_{\mu}(\mathbf{q})=z_{\mu}^{(0)}(\hat{\mathbf{q}})+(i q) z_{\mu}^{(1)}(\hat{\mathbf{q}})+(i q)^{2} z_{\mu}^{(2)}(\hat{\mathbf{q}})+\cdots
\end{aligned}
$$

From Eqs. (3) and (13) it follows that

$$
\sum_{i} a_{i} \Omega_{i j}=0
$$

In other words, the collisional invariants $a$ are left zero eigenvectors of $\Omega$. The dimensionality of the null space of $\Omega$ is equal to the number of collisional invariants: one for diffusive models, and $d+1$ for athermal (without energy conservation) fluid-type models. From Eq. (27) we conclude that for $\mathbf{q}=\mathbf{0}$ the left zero eigenvectors of the propagator are $\psi_{\mu}(\mathbf{0})=a$ with $z_{\mu}(\mathbf{0})=0$. These eigenmodes $\mu$, associated with local conservation laws, are called slow or hydrodynamic modes. It will be shown below that only pairs $\mu \nu$ of slow modes are responsible for singularities (here a discontinuity or anisotropy at $\mathbf{q}=\mathbf{0}$ ) in the $\mathbf{q}$ dependence of the susceptibilities, and hence for the existence of algebraic decay of the pair correlation function.

By expanding Eq. (23) in powers of (iq) we obtain the following hierarchy of equations for the left zero eigenvectors:

$$
\begin{gathered}
\Omega^{T} \psi_{\mu}^{(0)}=0, \\
\Omega^{T} \psi_{\mu}^{(1)}=\left(c_{\ell}+z_{\mu}^{(1)}\right) \psi_{\mu}^{(0)}, \\
\Omega^{T} \psi_{\mu}^{(2)}=\left(c_{\ell}+z_{\mu}^{(1)}\right) \psi_{\mu}^{(1)}+\left[z_{\mu}^{(2)}+\frac{1}{2}\left(c_{\ell}+z_{\mu}^{(1)}\right)^{2}\right] \psi_{\mu}^{(0)},
\end{gathered}
$$

where $\Omega^{T}$ is the transpose of $\Omega$, and $c_{\ell i}=\hat{\mathbf{q}} \cdot \mathbf{c}_{i}$. Similar equations hold for the right zero eigenvectors, but with $\Omega^{T}$ replaced by $\Omega$. The biorthonormality condition (24) must also holds to all powers of $(i q)$, which yields

$$
\begin{gathered}
\left\langle\psi_{\mu}^{(0)} \mid \widetilde{\psi}_{\nu}^{(0)}\right\rangle=\delta_{\mu \nu}, \\
\left\langle\psi_{\mu}^{(0)} \mid \widetilde{\psi}_{\nu}^{(1)}\right\rangle+\left\langle\psi_{\mu}^{(0)}\left|c_{\curlywedge}\right| \widetilde{\psi}_{\nu}^{(0)}\right\rangle+\left\langle\psi_{\mu}^{(1)} \mid \widetilde{\psi}_{\nu}^{(0)}\right\rangle=0,
\end{gathered}
$$

etc. Note that if $\Omega_{i j}$ is symmetric so that $\Omega^{T}=\Omega$, then $\psi_{\mu}(\mathbf{q})$ and $\widetilde{\psi}_{\mu}(\mathbf{q})$ are equal, up to a normalization factor. The perturbation equations $(28 \mathrm{a})-(28 \mathrm{c})$ have the general form $\Omega^{T} \psi_{\mu}^{(n)}=I_{\mu}^{(n)}$, where the inhomogeneous term $I_{\mu}^{(n)}$ depends on the unknown eigenvalue $z_{\mu}^{(n)}$. As the matrix $\Omega^{T}$ has left zero eigenvectors $\widetilde{\psi}_{\mu}^{(0)}$, it is required that

$$
0=\left\langle\widetilde{\psi}_{\nu}^{(0)} \mid \Omega^{T} \psi_{\mu}^{(n)}\right\rangle=\left\langle\widetilde{\psi}_{\nu}^{(0)} \mid I_{\mu}^{(n)}\right\rangle
$$

for all slow modes $\nu$. Solving these equations for $z_{\mu}^{(n)}$ enables us to determine the eigenvalues perturbatively. 


\section{E. Algebraic correlations}

Once $\Delta \chi_{a}(\mathbf{q})$ is calculated, Fourier inversion of Eq. (18) enables us to calculate the spatial correlation functions. In the limit of large system size we can make the continuum approximation,

$$
\frac{1}{V} \sum_{\mathbf{q}} \rightarrow \frac{v_{0}}{(2 \pi)^{d}} \int_{\mathrm{BZ}} d \mathbf{q}
$$

Here $v_{0}$ is the volume of a unit cell in the lattice $\left(v_{0}=\frac{1}{2} \sqrt{3}\right.$ for a triangular lattice; $v_{0}=1$ for a square or cubic lattice), and the $\mathbf{q}$ integration extends over the first Brillouin zone. The excess correlation function is then given by

$$
\mathcal{C}_{a}(\mathbf{r})=\frac{v_{0}}{(2 \pi)^{d}} \int_{\mathrm{BZ}} d \mathbf{q} e^{i \mathbf{q} \cdot \mathbf{r}} \Delta \chi_{a}(\mathbf{q})
$$

Combining Eqs. (25) and (32) we have

$$
\mathcal{C}_{a}(\mathbf{r})=\mathcal{C}_{a}^{(0)}(\mathbf{r})+\mathcal{C}_{a}^{(2)}(\mathbf{r})+\mathcal{C}_{a}^{(4)}(\mathbf{r})+\cdots
$$

Consider the contribution of the $O\left(q^{m}\right)$ term in Eq. (25) to $\mathcal{C}_{a}(\mathbf{r})$

$$
\mathcal{C}_{a}^{(m)}(\mathbf{r})=\frac{v_{0}}{(2 \pi)^{d}} \int_{\mathrm{BZ}} d \mathbf{q} q^{m} e^{i \mathbf{q} \cdot \mathbf{r}} \Delta \chi_{a}^{(m)}(\hat{\mathbf{q}})
$$

If $\Delta \chi_{a}^{(m)}(\hat{\mathbf{q}})=\Delta \chi_{a}^{(m)}$ is isotropic, i.e., continuous at $\mathbf{q}=\mathbf{0}$, then the right hand side of Eq. (34) is essentially a representation of (the $m$ th derivative of) the Dirac $\delta$ function, and therefore all correlations are short ranged. The situation is very different when $\Delta \chi_{a}^{(m)}(\hat{\mathbf{q}})$ is anisotropic, i.e., it depends on $\hat{\mathbf{q}}$ as $\mathbf{q} \longrightarrow \mathbf{0}$. A rescaling of $\mathbf{q}$ in Eq. (34) then shows that

$$
\mathcal{C}_{a}^{(m)}(\mathbf{r}) \simeq \frac{v_{0}}{(2 \pi)^{d}} \frac{1}{r^{d+m}} \int_{R^{d}} d \mathbf{q} q^{m} e^{i \mathbf{q} \cdot \hat{\mathbf{r}}} \Delta \chi_{a}^{(m)}(\hat{\mathbf{q}})
$$

where $\hat{\mathbf{r}}=\mathbf{r} /|\mathbf{r}|$. Therefore for large $r$ the pair correlation function $\mathcal{G}_{a}(\mathbf{r})$ behaves as

$$
\mathcal{G}_{a}(\mathbf{r}) \simeq \frac{A(\hat{\mathbf{r}})}{r^{d+m}}
$$

with a coefficient $A(\hat{\mathbf{r}})$ that depends on the direction of $\mathbf{r}$. The value of $m$ is determined by the first anisotropic term in the expansion (25) of the susceptibility. The amplitude $A(\hat{\mathbf{r}})$ can be calculated from the microscopic definition of the model by performing the Fourier integral in Eq. (35).

In the remainder of this paper we determine $m$ and calculate $A(\hat{\mathbf{r}})$ for two different models: (i) interacting random walkers on the square lattice with an anisotropic transition matrix $A_{s \sigma}$ yielding spatial density-density correlations of type $1 / r^{2}$ or $1 / r^{4}$, depending on whether or not the symmetry between $x$ and $y$ directions is broken, and (ii) a fluid-type model on a triangular lattice with spatial correlations of type $1 / r^{2}$ in the momentum density.

\section{INTERACTING RANDOM WALKERS WITH $x-y$ ANISOTROPY}

In this section we discuss a LGA for interacting random walkers on the square lattice. The collision rules of the model break the symmetry between the $x$ and $y$ directions. We choose a model that is still invariant under reflections in both the $x$ and $y$ axes, so that no average particle drift occurs. Collision rules that break the $x-y$ symmetry are most easily formulated in terms of the particle flux $\mathbf{J}(s)$ corresponding to a state $s$,

$$
\mathbf{J}(s)=\sum_{i} \mathbf{c}_{i} s_{i}
$$

We choose the matrix of transition probabilities as

$$
A_{s \sigma}=\frac{1}{Z(s)} \exp [\mathbf{J}(s) \cdot \mathbf{M} \cdot \mathbf{J}(\sigma)] \delta(\rho(s), \rho(\sigma)),
$$

where $Z(s)$ is a normalization constant,

$$
Z(s)=\sum_{\sigma} \exp [\mathbf{J}(s) \cdot \mathbf{M} \cdot \mathbf{J}(\sigma)] \delta(\rho(s), \rho(\sigma))
$$

and $\mathbf{M}$ is a diagonal matrix,

$$
\mathbf{M}=\left(\begin{array}{cc}
\beta_{x} & 0 \\
0 & \beta_{y}
\end{array}\right) .
$$

If $\beta_{x}=\beta_{y}=0$ then the detailed-balance condition is satisfied, and a completely factorized equilibrium state exists. For all other choices of $\beta_{x}$ and $\beta_{y}-$ positive or negative - the density-density correlations in the correlated equilibrium state decay algebraically. In the special case $\beta_{x}=\beta_{y} \neq 0$ the model has the complete symmetry of the underlying lattice. This case will be discussed in the next section. In the remainder of this section we show that when $\beta_{x} \neq \beta_{y}$ the correlations are of type $1 / r^{2}$. We derive an analytical expression for the amplitude, for the specific interacting random walker model defined by Eqs. (37)-(40).

The system of interacting random walkers (IRW's) on a (bipartite) square lattice, which make a move at each time step, consists in fact of two totally independent subsystems: the IRW's initially on the even sublattice $\mathcal{L}_{+}$and those initially on the odd sublattice $\mathcal{L}_{-}$. In a single time step all particles on $\mathcal{L}_{+}$move to $\mathcal{L}_{-}$and vice versa. Therefore equaltime correlations can only exist between particles at positions $\mathbf{r}$ and $\mathbf{r}^{\prime}$ on the same sublattice. Consequently, the difference $\mathbf{r}-\mathbf{r}^{\prime}$ always belongs to the even sublattice, so that $\mathcal{G}(\mathbf{r}) \equiv 0$ for $\mathbf{r} \in \mathcal{L}_{-}$, and $\mathcal{G}(\mathbf{r})$ is possibly nonvanishing for $\mathbf{r} \in \mathcal{L}_{+}$.

The above features of the bipartite square lattice are contained in the mode coupling formula (22) through the existence of two slow modes, both contributing to the long-range part of the pair correlation function. Let $N_{+}(t)$ and $N_{-}(t)$ denote the total number of particles at time $t$ on $\mathcal{L}_{+}$and $\mathcal{L}_{-}$, respectively; then their difference oscillates in time, and $N_{\theta}=(-)^{t}\left[N_{+}(t)-N_{-}(t)\right]$ is a conserved quantity, just like the total number of particles $N=N_{+}(t)+N_{-}(t)$. The slow mode corresponding to the conservation of $N_{\theta}$ is called the 
staggered diffusive mode; the one corresponding to the conservation of $N$ is the usual diffusive mode.

The regular diffusive mode has a relaxation rate that for small wave number behaves as [see Eq. (A22) of Appendix A)]

$$
z_{D}(\mathbf{q}) \simeq-q^{2} z_{2}(\hat{\mathbf{q}})=-\left(D_{x} q_{x}^{2}+D_{y} q_{y}^{2}\right),
$$

with diffusion coefficients in the $x$ and $y$ directions given by Eq. (A23). To leading order the excess susceptibility $\Delta \chi(\mathbf{q}) \equiv \Delta \chi_{\rho}(\mathbf{q})$ contains a contribution from a pair of diffusive modes, i.e.,

$$
\begin{aligned}
\Delta \chi(\mathbf{q})= & \left\langle 11 \mid \widetilde{\psi}_{D}(\mathbf{q}) \widetilde{\psi}_{D}(-\mathbf{q})\right\rangle\left(\frac{1}{1-e^{2 z_{D}(\mathbf{q})}}\right) \\
& \times\left\langle\psi_{D}(\mathbf{q}) \psi_{D}(-\mathbf{q}) \mid E\right\rangle .
\end{aligned}
$$

In Appendix A the left and right diffusive eigenvectors $\psi_{D}(\mathbf{q})$ and $\widetilde{\psi}_{D}(\mathbf{q})$ are calculated using perturbation theory. For small $q$ the amplitude factors in Eq. (42) are calculated in Eqs. (A18) and (A27) with the result

$$
\begin{gathered}
\left\langle 11 \mid \widetilde{\psi}_{D}(\mathbf{q}) \widetilde{\psi}_{D}(-\mathbf{q})\right\rangle=1, \\
\left\langle\psi_{D}(\mathbf{q}) \psi_{D}(-\mathbf{q}) \mid E\right\rangle=2\left(B_{x} q_{x}^{2}+B_{y} q_{y}^{2}\right) .
\end{gathered}
$$

The factor involving the eigenvalue $z_{D}$ is given by

$$
1-e^{2 z_{D}(\mathbf{q})} \simeq 2\left(D_{x} q_{x}^{2}+D_{y} q_{y}^{2}\right) .
$$

In Eq. (A25) of Appendix A the coefficients $B_{\alpha}$ are given explicitly. In the majority of publications on driven diffusive systems $[2,4,5]$, the transport coefficients $D_{\alpha}$ and the coefficients $B_{\alpha}$ - which in the phenomenological description represent the noise strength of the fluctuating force in the Langevin equation - are simply phenomenological input in the theory. In the present paper both sets of coefficients $D_{\alpha}$ and $B_{\alpha}$ are calculated from the microscopic definition of the model.

From Eqs. (42)-(44) it can be seen that the limit $\Delta \chi^{(0)}(\hat{\mathbf{q}}) \equiv \lim _{q \rightarrow 0} \Delta \chi(\mathbf{q})$ exists and that the dominant part of this contribution to the excess susceptibility is given by

$$
\Delta \chi_{D}^{(0)}(\hat{\mathbf{q}})=\frac{B_{x} \hat{q}_{x}^{2}+B_{y} \hat{q}_{y}^{2}}{D_{x} \hat{q}_{x}^{2}+D_{y} \hat{q}_{y}^{2}}
$$

Inverse Fourier transformation yields the contribution $\mathcal{G}_{D}(\mathbf{r})$ of the two diffusive modes to the large- $r$ behavior of the pair correlation function,

$$
\mathcal{G}_{D}(\mathbf{r}) \simeq\left(\frac{D_{x} B_{y}-D_{y} B_{x}}{2 \pi \sqrt{D_{x} D_{y}}}\right) \frac{D_{y} x^{2}-D_{x} y^{2}}{\left(D_{y} x^{2}+D_{x} y^{2}\right)^{2}} .
$$

However, the staggered slow mode also contributes to the excess susceptibility. It occurs at the wave vector $\boldsymbol{\pi}=(\pi, \pi)$, and is intimately related to the diffusive mode $\mu=D \quad$ occurring at $\mathbf{q}=\mathbf{0}$. We have $\Gamma(\mathbf{q}+\boldsymbol{\pi})$ $=e^{-i \boldsymbol{\pi} \cdot \mathbf{c}_{i}} \Gamma(\mathbf{q})=-\Gamma(\mathbf{q})$, where $\quad \Gamma(\mathbf{q})=e^{i \mathbf{q} \cdot \mathbf{c}}(1+\Omega) \quad$ is the one-step propagator with eigenvalue $e^{z_{D}(\mathbf{q})}$. Then $z_{D}(\mathbf{q}+\boldsymbol{\pi})=z_{D}(\mathbf{q})+i \pi, \psi_{D}(\mathbf{q}+\boldsymbol{\pi})=\psi_{D}(\mathbf{q})$, and $\widetilde{\psi}_{D}(\mathbf{q}+\boldsymbol{\pi})$ $=\widetilde{\psi}_{D}(\mathbf{q})$. It follows that $\Delta \chi(\mathbf{q}+\boldsymbol{\pi})=\Delta \chi(\mathbf{q})$. The staggered

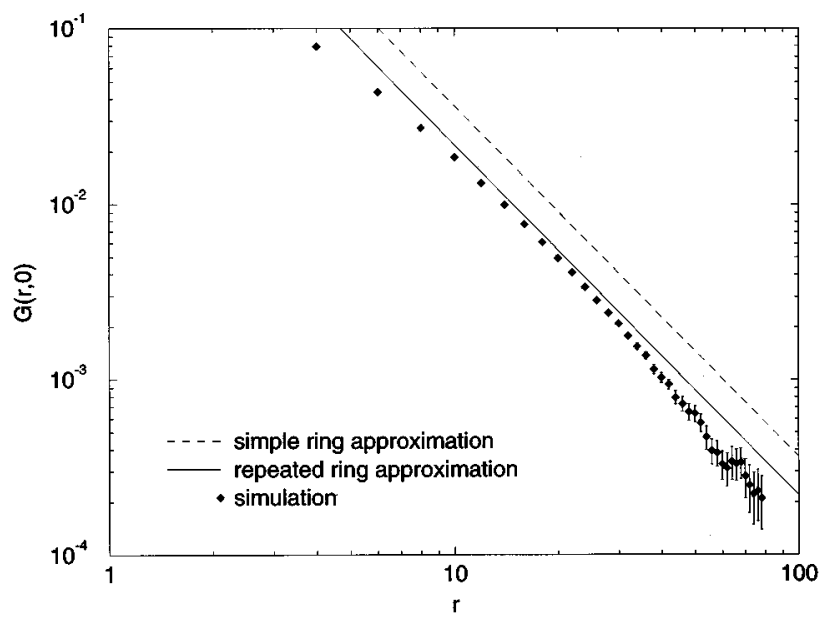

FIG. 1. Anisotropic interacting random walker model. Pair correlation function $\mathcal{G}(\mathbf{r})$, at even $r$ values, with $\mathbf{r}=(r, 0)$ along the $x$ axis, for interacting random walkers on a square lattice with interactions that break the symmetry between the $x$ and $y$ axes. For $r=$ odd the pair correlation function vanishes. The average density per velocity channel is $f=1 / 2$, and the model parameters are $\beta_{x}=1$ and $\beta_{y}=3$. Symbols with error bars indicate simulation results for a system of $512^{2}$ nodes, with an equilibration time of $T_{\text {eq }}=10^{4}$ time steps. The lines denote the asymptotic algebraic tail $\sim 1 / r^{2}$, as predicted by ring kinetic theory in the simple (dashed line) and repeated ring approximation (solid line).

mode at $\mathbf{q}=\boldsymbol{\pi}$ gives a contribution to the pair correlation function equal to $e^{-i \pi \cdot \mathbf{r}} \mathcal{G}_{D}(\mathbf{r})$, so that the final result for the pair correlation reads

$\mathcal{G}(\mathbf{r})=\left(1+e^{-i \boldsymbol{\pi} \cdot \mathbf{r}}\right) \mathcal{G}_{D}(\mathbf{r})=\left\{\begin{array}{lll}2 \mathcal{G}_{D}(\mathbf{r}), & x+y & \text { even } \\ 0, & x+y & \text { odd }\end{array}\right.$

To test the accuracy of our prediction we have performed computer simulations for $\beta_{x}=1$ and $\beta_{y}=3$ at the half-filled lattice, where $f_{i}=\frac{1}{2}$ for all four velocity directions. To obtain numerical values for the source matrix $E_{i j}$ in the repeated ring approximation, Eq. (12), we determined the on-site correlations $\mathcal{C}_{i j}$ using the methods of Ref. [11].

Figure 1 shows a comparison between simulation data and the analytical prediction of the large- $r$ behavior. The repeated ring theory agrees well with the simulation values over the range $r \in[10,50]$. For large $r$ there is a systematic deviation of the simulation data that is a result of the slow diffusive equilibration on large spatial scales, according to $r^{2} \sim D t$, where $D$ is the smaller of the two diffusion constants $D_{x}$ and $D_{y}$.

\section{INTERACTING RANDOM WALKERS WITH SQUARE LATTICE SYMMETRY}

In this section we discuss the behavior of general diffusive LGA's with collision rules that obey all symmetries of the underlying lattice, but violate detailed balance. An example of such a LGA is the model of the previous section in the special case $\beta_{x}=\beta_{y} \neq 0$. The collision rules then obey all symmetries of the square lattice (reflection in $x$ or $y$ axis, and rotation over multiples of $90^{\circ}$ ), which implies that second 
rank tensors are isotropic. Therefore the anisotropy giving rise to $1 / r^{2}$ correlation for $\beta_{x} \neq \beta_{y}$, as discussed in the previous section, is now absent. However, on the square lattice tensors of rank 4 contain anisotropic parts. In what follows we explain how this anisotropy gives rise to correlations decaying as $1 / r^{4}$.

There are again two slow modes: the usual diffusion mode and the staggered diffusive mode. The corresponding eigenvalue $z_{D}(\mathbf{q})$ of the diffusion mode has the form

$$
z_{D}(\mathbf{q})=-D q^{2}+D_{2}(\hat{\mathbf{q}}) q^{4}+\cdots .
$$

All odd terms vanish because of reflection symmetry. On the square lattice, the so-called super-Burnett coefficient $D_{2}(\hat{\mathbf{q}})$ depends on the direction $\hat{\mathbf{q}}$ of the wave vector $\mathbf{q}$. It contains an anisotropic term equal to $-2 D_{2}^{\prime} \hat{q}_{x}^{2} \hat{q}_{y}^{2}$ on account of Eq. (A30).

To calculate the excess correlation function in Eq. (42) we analyze its separate factors. The factor containing the eigenvalue $z_{D}(\mathbf{q})$ behaves for small $\mathbf{q}$ as

$$
\frac{1}{1-e^{2 z_{D}(\mathbf{q})}}=\frac{1}{2 D q^{2}}\left\{1+q^{2}\left(\frac{D_{2}(\hat{\mathbf{q}})}{D}+D\right)+\cdots\right\} .
$$

The first factor in Eq. (42) equals unity for small $\mathbf{q}$ [see Eq. (43)]. The last one behaves as

$$
\left\langle\psi_{D}(\mathbf{q}) \psi_{D}(-\mathbf{q}) \mid E\right\rangle=2 B q^{2}+2 B_{2}(\hat{\mathbf{q}}) q^{4}+\cdots,
$$

where the isotropic $B$ and the anisotropic $B_{2}(\hat{\mathbf{q}})$ are calculated in Eqs. (A33) and (A34); $B_{2}(\hat{\mathbf{q}})$ contains an anisotropic term $-2 B_{2}^{\prime} \hat{q}_{x}^{2} \hat{q}_{y}^{2}$. Isotropic terms do not contribute to the algebraic correlations. After collecting terms, the dominant anisotropic contribution of the two diffusion modes to the susceptibility becomes

$$
\begin{aligned}
\Delta \chi_{D}(\hat{\mathbf{q}}) & \simeq \frac{B}{D}\left(\frac{D_{2}(\hat{\mathbf{q}})}{D}+\frac{B_{2}(\hat{\mathbf{q}})}{B}\right) q^{2} \\
& =-\frac{2 B}{D}\left(\frac{D_{2}^{\prime}}{D}+\frac{B_{2}^{\prime}}{B}\right) \hat{q}_{x}^{2} \hat{q}_{y}^{2} q^{2} \\
& \equiv-A \hat{q}_{x}^{2} \hat{q}_{y}^{2} q^{2} .
\end{aligned}
$$

The large- $r$ behavior of the inverse Fourier transform of the anisotropic part of Eq. (51) is given by

$$
\begin{aligned}
\mathcal{G}(\mathbf{r}) & \simeq-\left(1+e^{-i \boldsymbol{\pi} \cdot \mathbf{r}}\right) \frac{A}{(2 \pi)^{2} r^{4}} \int d \mathbf{q} q^{2} e^{i \mathbf{q} \cdot \hat{\mathbf{r}}} \hat{q}_{x}^{2} \hat{q}_{y}^{2} \\
& =\left(1+e^{-i \pi \cdot \mathbf{r}}\right) \frac{3 A}{\pi r^{4}}\left[\left(\frac{x}{r}\right)^{2}-\left(\frac{y}{r}\right)^{2}\right]^{2}
\end{aligned}
$$

The factor $\left(1+e^{-i \pi \cdot \mathbf{r}}\right)$ accounts for the contribution of the staggered diffusive modes, as explained below Eq. (46). This result represents the long-range behavior of the pair correlation function of interacting random walkers with interactions having the full square lattice symmetry. The important conclusion of this calculation is that the amplitude of the $1 / r^{4}$ tail is nonzero for general choices of $\beta_{x}=\beta_{y} \neq 0$. Thus the model provides an explicit microscopic realization of the scenario that was discussed in the context of the Langevin equation by Grinstein et al. [5]. The $1 / r^{4}$ tail is much weaker than the $1 / r^{2}$ tail discussed in the previous section, and therefore a comparison with computer simulations would require a numerical effort that is beyond the scope of this paper.

\section{FLUID-TYPE MODEL}

In this section we study the spatial correlation functions $\mathcal{G}_{\alpha \beta}(\mathbf{0})=\left\langle g_{\alpha}(\mathbf{r}) g_{\beta}(\mathbf{r})\right\rangle$ of the momentum densities, with $\alpha, \beta=\{x, y\}$, in a seven-bit LGA fluid defined on a triangular lattice, which allows for a rest particle state (see Sec. II A) and violates detailed balance.

The susceptibility $\chi_{\alpha \beta}(\mathbf{q})$ is defined as the Fourier transform of the correlation function $\mathcal{G}_{\alpha \beta}(\mathbf{r})=\left\langle g_{\alpha}(\mathbf{r}) g_{\beta}(\mathbf{0})\right\rangle$ with $\alpha, \beta=x, y$. We decompose $\chi_{\alpha \beta}(\mathbf{q})$ into a longitudinal and a transverse part, as

$$
\chi_{\alpha \beta}(\mathbf{q})=\hat{\mathbf{q}}_{\alpha} \hat{\mathbf{q}}_{\beta} \chi \lambda(\mathbf{q})+\left(\delta_{\alpha \beta}-\hat{\mathbf{q}}_{\alpha} \hat{\mathbf{q}}_{\beta}\right) \chi_{\perp}(\mathbf{q}),
$$

where

$$
\chi_{\curlywedge}(\mathbf{q})=\left\langle g_{\ell}(\mathbf{q}) g_{\curlywedge}(-\mathbf{q})\right\rangle
$$

and

$$
\chi_{\perp}(\mathbf{q})=\left\langle g_{\perp}(\mathbf{q}) g_{\perp}(-\mathbf{q})\right\rangle
$$

are scalar fields with identical diagonal parts given by

$$
\chi_{\ell}^{d}=\chi_{\perp}^{d}=3 f(1-f)
$$

on account of Eq. (5). The excess parts $\Delta \chi_{\ell}(\mathbf{q})$ and $\Delta \chi_{\perp}(\mathbf{q})$, given by Eq. (22) with $a=c_{/}$and $a=c_{\perp}$, respectively, are, in general, different. As we will argue below, the limits for $\mathbf{q} \rightarrow \mathbf{0}$ of $\Delta \chi_{,}(\mathbf{q})$ and $\Delta \chi_{\perp}(\mathbf{q})$, denoted by $\Delta \chi_{\ell}$ and $\Delta \chi_{\perp}$, are nonvanishing. If $\Delta \chi_{\ell} \neq \Delta \chi_{\perp}$ then $\chi_{\alpha \beta}^{(0)}(\hat{\mathbf{q}})=\lim _{q \rightarrow 0} \chi_{\alpha \beta}(\mathbf{q})$ is anisotropic at $\mathbf{q}=\mathbf{0}$ and therefore $\mathcal{G}_{\alpha \beta}(\mathbf{r}) \sim 1 / r^{2}$ for large $r$.

To determine the dominant long-range part of $\mathcal{G}_{\alpha \beta}(\mathbf{r})$ we need to know, according to Eq. (22), the right and left hydrodynamic modes $\left\{\psi_{\mu}(\mathbf{q}), \widetilde{\psi}_{\mu}(\mathbf{q})\right\}$ and eigenvalues $z_{\mu}(\mathbf{q})$ of the lattice Boltzmann equation, defined through Eq. (23). The collisional invariants in this model are $a_{\alpha}=\left\{1, c_{x}, c_{y}\right\}$ or equivalently $a_{\alpha}=\left\{a_{\rho}, a_{\ell}, a_{\perp}\right\}=\left\{1, c_{\ell}, c_{\perp}\right\}$, where longitudinal $(\ell)$ and transverse $(\perp)$ refer to the q direction. The set $\left\{a_{\alpha}\right\}$ are the zero left eigenvectors of the collision operator $\Omega$, and $\widetilde{a}_{\alpha}$ are the corresponding zero right eigenvectors, i.e., $\Omega^{T} a_{\alpha}=0$ and $\Omega \widetilde{a}_{\alpha}=0$. The left and right eigenvectors form a biorthogonal set, i.e., $\left\langle a_{\alpha} \mid \widetilde{a}_{\beta}\right\rangle=\delta_{\alpha \beta}$.

Symmetry properties and the complete set of eigenvectors and eigenvalues are discussed in Appendix B, and summarized in Table II, where $u_{0}=a_{\rho}, u_{2}=a_{\ell}$, and $u_{3}=a_{\perp}$. It is convenient for what follows to show how eigenvalues and eigenvectors transform under the inversion $\mathbf{q} \rightarrow-\mathbf{q}$. We first observe that $z_{\mu}(\mathbf{q})=z_{\mu}^{*}(-\mathbf{q})$ for all modes. Moreover, complex conjugation of Eq. (22) shows

$$
\begin{aligned}
& \psi_{\sigma}^{*}(\mathbf{q})=\psi_{-\sigma}(-\mathbf{q}), \quad z_{\sigma}^{*}(\mathbf{q})=z_{-\sigma}(\mathbf{q}), \\
& \psi_{\perp}^{*}(\mathbf{q})=-\psi_{\perp}(-\mathbf{q}), \quad z_{\perp}^{*}(\mathbf{q})=z_{\perp}(\mathbf{q}),
\end{aligned}
$$

and the same relations with $\psi_{\mu} \rightarrow \widetilde{\psi}_{\mu}$. 
For small $q$ the shear mode or transverse momentum mode $(\mu=\perp)$ is

$$
\psi_{\perp}^{(0)}(\mathbf{q})=a_{\perp}, \quad \widetilde{\psi}_{\perp}^{(0)}(\mathbf{q})=\widetilde{a}_{\perp}, \quad z_{\perp}(\mathbf{q})=-\nu q^{2},
$$

and the sound modes $(\mu=\sigma= \pm)$ are

$$
\begin{gathered}
\psi_{\sigma}^{(0)}(\mathbf{q})=a_{\ell}+\sigma v_{s} a_{\rho}, \quad \widetilde{\psi}_{\sigma}^{(0)}(\mathbf{q})=\frac{1}{2}\left(\widetilde{a}_{\perp}+\frac{\sigma}{v_{s}} \tilde{a}_{\rho}\right), \\
z_{\sigma}(\mathbf{q})=-i q \sigma v_{s}-\Gamma q^{2} .
\end{gathered}
$$

The vectors $\widetilde{a_{\alpha}}(\alpha=\rho, \ell, \perp)$ are also given in Table II. The shear viscosity $\nu$, the speed of sound $v_{s}$, and the sound damping constant $\Gamma$ can be expressed in terms of matrix elements $\Omega_{i j}$ of the collision operator, as shown in Appendix B 3, where the higher order coefficients in the $q$ expansion, $\psi_{\mu}^{(n)}$, are also determined.

We start with the transverse susceptibility in Eq. (22) and observe that only the pair $(\mu \nu)=(\perp)$ has a nonvanishing overlap for small $q$, i.e., $\left\langle c_{\perp} c_{\perp} \mid \widetilde{\psi}_{\perp}(\mathbf{q}) \widetilde{\psi}_{\perp}(-\mathbf{q})\right\rangle \simeq-1$ for small q. The excess susceptibility then has the form

$$
\Delta_{\perp}(\mathbf{q}) \simeq-\frac{1}{2 \nu q^{2}}\left\langle\psi_{\perp}(\mathbf{q})|E| \psi_{\perp}(-\mathbf{q})\right\rangle .
$$

Inserting the $q$ expansion (26) for $\psi_{\perp}$, and using the relations (55) and $\left\langle a_{\alpha}|E| a_{\beta}\right\rangle=0$ [see Eq. (14)], the dominant small- $q$ term in Eq. (58) is then

$$
\Delta \chi_{\perp}(\mathbf{q})=\frac{1}{2 \nu}\left[\left\langle\psi_{\perp}^{(1)}|E| \psi_{\perp}^{(1)}\right\rangle-2\left\langle\psi_{\perp}^{(0)}|E| \psi_{\perp}^{(2)}\right\rangle\right] .
$$

These terms are evaluated in Eq. (B24) of Appendix B 3 with the result

$$
\Delta \chi_{\perp}(\mathbf{q})=\frac{3}{8 \nu}\left(\frac{\epsilon_{4}}{\omega_{4}^{2}}\right) .
$$

The eigenvalues $-\omega_{4}$ and $\epsilon_{4}$ of $\Omega$ and $E$ are calculated in Appendixes B 1 and B 2, and listed in Tables II and III.

Next consider the longitudinal susceptibility in Eq. (22), where only sound modes $(\mu \nu)=\left(\sigma, \sigma^{\prime}\right)$ give a nonvanishing contribution for small $\mathbf{q}$, i.e.,

$$
\left\langle c_{\ell} c / \widetilde{\psi}_{\sigma}(\mathbf{q}) \widetilde{\psi}_{\sigma^{\prime}}(-\mathbf{q})\right\rangle \simeq \frac{1}{4},
$$

and the excess susceptibility becomes

$$
\begin{aligned}
\Delta \chi /(\mathbf{q})= & \frac{1}{4} \sum_{\sigma, \sigma^{\prime}}\left[i q\left(\sigma+\sigma^{\prime}\right) v_{s}+2 \Gamma q^{2}\right]^{-1} \\
& \times\left\langle\psi_{\sigma}(\mathbf{q})|E| \psi_{-\sigma^{\prime}}^{*}(\mathbf{q})\right\rangle .
\end{aligned}
$$

For parallel sound modes, $\sigma^{\prime}=\sigma$, the denominator yields $2 i q \sigma v_{s}$ for small $q$, and the last factor in Eq. (62) yields

$$
\begin{aligned}
\left\langle\psi_{\sigma}(\mathbf{q})|E| \psi_{-\sigma}^{*}(\mathbf{q})\right\rangle & \simeq i q\left[\left\langle\psi_{\sigma}^{(0)}|E| \psi_{-\sigma}^{(1)}\right\rangle+\left\langle\psi_{\sigma}^{(1)}|E| \psi_{-\sigma}^{(0)}\right\rangle\right] \\
& =2 i q \sigma v_{s} \mathcal{E}_{10} / \omega_{1} .
\end{aligned}
$$

The latter equality is derived in Eq. (B25); the coefficient $\mathcal{E}_{10}$, defined in Eq. (B26), is calculated in Eq. (B33) in terms of the $E_{i j}$ 's defined in Eqs. (9) and (12). For opposite sound modes, $\sigma^{\prime}=-\sigma$, the denominator becomes $2 \Gamma q^{2}$ and the latter factor in Eq. (62) yields

$$
\begin{aligned}
\left\langle\psi_{\sigma}(\mathbf{q})\right| & E\left|\psi_{\sigma}^{*}(\mathbf{q})\right\rangle \\
& \simeq q^{2}\left[\left\langle\psi_{\sigma}^{(1)}|E| \psi_{\sigma}^{(1)}\right\rangle-2\left\langle\psi_{\sigma}^{(0)}|E| \psi_{\sigma}^{(2)}\right\rangle\right] \\
& =q^{2}\left\{\frac{3 \epsilon_{4}}{4 \omega_{4}^{2}}+\frac{\mathcal{E}_{11}}{\omega_{1}^{2}}-\frac{2 \mathcal{E}_{10}}{\omega_{1}}\left[\Gamma+v_{s}^{2}\left(\frac{1}{2}-\frac{1}{\omega_{1}}\right)\right]\right\} .
\end{aligned}
$$

Here the latter equality is derived in Eqs. (B27)-(B31), and the coefficient $\mathcal{E}_{11}$ is calculated in Eq. (B33) in terms of $E_{i j}$ 's. Combining Eqs. (62)-(64) yields the final result,

$$
\Delta \chi_{\ell}=\frac{3 \epsilon_{4}}{16 \Gamma \omega_{4}^{2}}+\frac{E_{00}}{16 \Gamma \omega_{1}^{2}}+\frac{E_{00}+6 E_{10}}{8 \Gamma \omega_{1}}\left(v_{s}^{2}-\frac{1}{\omega_{1}}\right) .
$$

In the case of the simple ring approximation, defined in Eq. (9) as $E_{i j}=\Omega_{i j}^{2,0}$, the above expressions simplify considerably because all diagonal elements are vanishing. It follows from Eqs. (7) and (9) that

$$
\begin{aligned}
E_{i i} & =\Omega_{i i}^{2,0}=\left(1-2 f_{i}\right) \sum_{s \sigma}\left(\sigma_{i}-s_{i}\right) A_{s \sigma} F(s) \\
& =\left(1-2 f_{i}\right) \Omega_{i}^{1,0}=0
\end{aligned}
$$

for all $i=0,1, \ldots, b$. We have used the relation $\left(\delta \sigma_{i}\right)^{2}=\left(\sigma_{i}-f_{i}\right)^{2}=\left(1-2 f_{i}\right) \sigma_{i}+f_{i}^{2}$, valid for Boolean variables $\sigma_{i}$. In this case the relevant eigenvalue in Eq. (B13) reduces to $\epsilon_{4}=-2 E_{13}$ and the excess longitudinal susceptibility becomes

$$
\Delta \chi_{\ell}=-\frac{3 E_{13}}{8 \Gamma \omega_{4}^{2}}+\frac{3 E_{10}}{4 \Gamma \omega_{1}}\left(v_{s}^{2}-\frac{1}{\omega_{1}}\right) .
$$

This simplification does not apply in the more general (repeated ring) case.

In general $\chi_{\ell}$ and $\chi_{\perp}$ are different, unless the collision rules satisfy detailed balance so that $E_{10}=E_{11}=E_{13}=0$. By inverse Fourier transformation of Eq. (53) we find that the asymptotic behavior of the correlation function is given by

$$
\mathcal{G}_{x x}(\mathbf{r})=-\mathcal{G}_{y y}(\mathbf{r})=\frac{\left(\chi_{\perp}-\chi_{l}\right) \sqrt{3}}{4 \pi}\left(\frac{x^{2}-y^{2}}{r^{4}}\right)
$$

and

$$
\mathcal{G}_{x y}(\mathbf{r})=\mathcal{G}_{y x}(\mathbf{r})=\frac{\left(\chi_{\perp}-\chi_{\ell}\right) \sqrt{3}}{4 \pi}\left(\frac{x y}{r^{4}}\right) .
$$

An equivalent statement, stressing the isotropy of the correlation functions, is that

$$
\mathcal{G}_{\ell}(r)=\frac{\left(\chi_{\perp}-\chi_{\ell}\right) \sqrt{3}}{4 \pi r^{2}},
$$

$\mathcal{G}_{\perp \perp}(r)=-\mathcal{G}_{\ell}(r)$, and $\mathcal{G}_{\perp}(r)=0$. The labels $\ell$ and $\perp$ here refer to the vector $\mathbf{r}$. In Eqs. (68)-(70) we may replace $\chi_{\ell}$ and $\chi_{\perp}$ by $\Delta \chi_{\ell}$ and $\Delta \chi_{\perp}$, respectively, on account of Eqs. (19) and (54). 


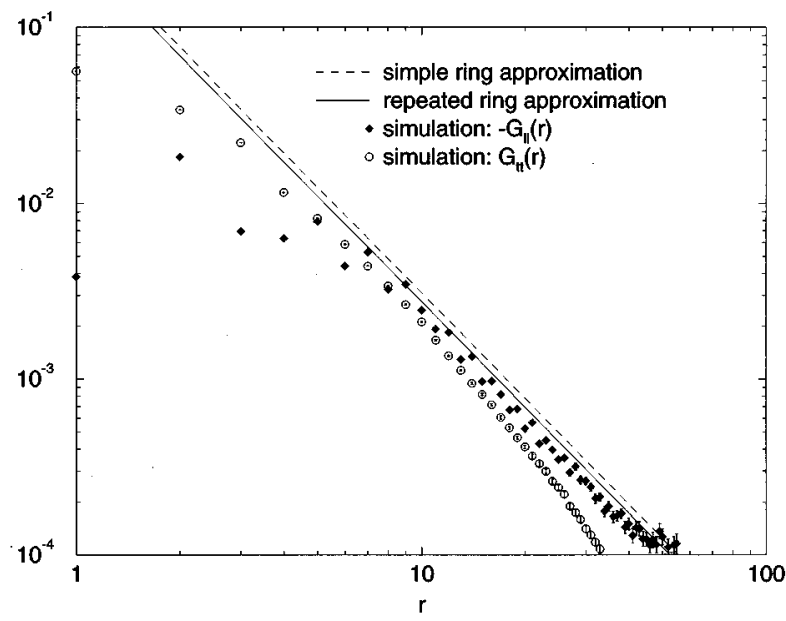

FIG. 2. Isotropic fluid-type model defined in Sec. V. Correlation function for the longitudinal, $\mathcal{G}_{\ell}(r)$, and transverse, $\mathcal{G}_{\perp \perp}(r)$, components of the momentum density. The average density is $f=1 / 2$ and the total momentum is zero. Symbols with error bars indicate simulation results for a system of $256^{2}$ nodes, with an equilibration time of $T_{\mathrm{eq}}=10^{4}$ time steps. The lines denote the asymptotic algebraic tail $\sim 1 / r^{2}$, as predicted by ring kinetic theory in the simple (dashed line) and repeated ring approximation (solid line).

We performed a computer simulation for the triangular lattice fluid-type LGA defined as model III in Fig. 1 of Ref. [14], where it was used to study tagged particle diffusion in a non-detailed-balance LGA fluid. Figure 2 shows a comparison between simulation results and the theoretical predictions for the amplitude of the algebraic tail. The statistics was improved by averaging $\mathcal{G}_{\ell}(r)$ and $\mathcal{G}_{\perp \perp}(r)$ over all directions. In particular when the repeated ring approximation is used, the agreement is quite satisfactory.

Although in the limit of long times $\mathcal{G}_{\ell}(r)$ and $\mathcal{G}_{\perp \perp}(r)$ are the same up to an overall sign, there is an interesting difference concerning the way in which equilibrium is reached. The buildup of $\mathcal{G}_{\ell}(r)$ is governed by traveling sound modes, for which $r \sim t$. The buildup of $\mathcal{G}_{\perp \perp}(r)$, however, involves the diffusive shear mode, so that $r^{2} \sim \nu t$. For the data shown in Fig. 2 the shear viscosity has the value $\nu \simeq 0.2$, so that the range over which $\mathcal{G}_{\perp \perp}(r)$ has equilibrated in $T_{\text {eq }}=10^{4}$ time steps is $\left(\nu T_{\text {eq }}\right)^{1 / 2} \simeq 45$ lattice spacings, in agreement with Fig. 2.

\section{DISCUSSION}

We have formulated a general ring kinetic theory for lattice gas automata, and used it to calculate the pair correlation function for conserved densities. These correlation functions have algebraic tails, $\mathcal{G}(\mathbf{r}) \simeq A(\hat{\mathbf{r}}) / r^{n}$, for large $r$. The exponent $n$ can be determined on the basis of symmetry considerations alone, using a conceptually simple phenomenological Langevin equation approach [2,5]. However, a theoretical estimate for the amplitude $A(\hat{\mathbf{r}})$ can only be obtained by approximately solving the kinetic equations that define the evolution in phase space, and analyzing the large- $r$ behavior of its stationary solution. This is exactly what we did in this paper.

To test the validity of our approach we performed com-
TABLE I. Symmetries on the square lattice.

\begin{tabular}{cccc}
\hline \hline$n$ & $u_{n}(\mathbf{c})$ & $x \leftrightarrow-x$ & $y \leftrightarrow-y$ \\
\hline 1 & 1 & + & + \\
2 & $c_{x}^{2}-c_{y}^{2}$ & + & + \\
3 & $c_{x}$ & - & + \\
4 & $c_{y}$ & + & - \\
\hline \hline
\end{tabular}

puter simulations for two different two-dimensional models, both violating the condition of semidetailed balance. First we considered a model of interacting random walkers with different diffusion coefficients in the $x$ and $y$ directions, exhibiting an algebraic decay of the density-density correlation function $\mathcal{G}_{\rho}(\mathbf{r})=\langle\delta \rho(\mathbf{r}) \delta \rho(\mathbf{0})\rangle \sim 1 / r^{2}$. The second model we considered was a fluid-type model in which the correlation function of momentum densities behaves as $\mathcal{G}_{\alpha \beta}(\mathbf{r})$ $=\left\langle g_{\alpha}(\mathbf{r}) g_{\beta}(0)\right\rangle \sim 1 / r^{2}$. In both cases we found good agreement between the simulated and theoretical values for the amplitude, in particular when we used the so-called repeated ring approximation, in which all pair correlation effects are taken into account in a self-consistent manner.

Most studies of nonequilibrium states using simple models so far have employed kinetic Ising models. Since lattice gas automata are easily implemented and analyzed, as well as flexible, they provide an attractive alternative. This holds in particular if one wishes to study fluid-type systems in which the momentum density is an additional conserved quantity. The algebraic momentum correlations discussed in Sec. V have to our knowledge not been observed before, either in computer simulations or in Langevin equation studies. It is an interesting question whether such correlations could be detected in real systems, e.g., in nonequilibrium states of molecular fluids or of granular media.

We expect that the techniques used here to analyze lattice gas automata can be extended to kinetic Ising models, since the latter constitute just a different class of cellular automata. This possibility is under investigation. So far there exists no microscopic theory providing the amplitude of algebraic spatial correlations in kinetic Ising models [4].

\section{APPENDIX A: INTERACTING RANDOM WALKERS}

\section{Structure of $\Omega$}

The right eigenvectors $\widetilde{u_{n}}$, the left eigenvectors $u_{n}$, and the corresponding eigenvalues $-\omega_{n}$ of the Boltzmann collision operator $\Omega$ are defined by

$$
\Omega \widetilde{u_{n}}=-\omega_{n} \widetilde{u_{n}}, \quad \Omega^{T} u_{n}=-\omega_{n} u_{n} .
$$

The eigenvectors are constructed solely on the basis of the square lattice symmetry, and are given by

$$
\begin{gathered}
1=(1,1,1,1), \\
c_{x}^{2}-c_{y}^{2}=(1,-1,1,-1), \\
c_{x}=(1,0,-1,0), \\
c_{y}=(0,1,0,-1) .
\end{gathered}
$$

Table I shows how these vectors behave under reflection in 
the $x$ and $y$ axis, respectively. There are three invariant subspaces, spanned by $\left\{1, c_{x}^{2}-c_{y}^{2}\right\}, c_{x}$, and $c_{y}$, respectively. Thus, on the basis of square symmetry alone, it can be seen that $c_{x}$ and $c_{y}$ are both left and right eigenvectors of $\Omega$ and $E$ (cf. Ref. [15]). The square symmetry implies that $\Omega_{i j}$ has only six independent elements, i.e.,

$$
\Omega=\left(\begin{array}{llll}
\Omega_{11} & \Omega_{12} & \Omega_{13} & \Omega_{12} \\
\Omega_{21} & \Omega_{22} & \Omega_{21} & \Omega_{24} \\
\Omega_{13} & \Omega_{12} & \Omega_{11} & \Omega_{12} \\
\Omega_{21} & \Omega_{24} & \Omega_{21} & \Omega_{22}
\end{array}\right) .
$$

Number conservation, expressed by $\langle 1| \Omega=0$, or explicitly,

$$
\Omega_{11}+2 \Omega_{21}+\Omega_{13}=\Omega_{22}+2 \Omega_{12}+\Omega_{24}=0,
$$

imposes two more relations, leaving only four independent elements. We easily obtain the following biorthonormal set of eigenvectors:

$$
\begin{gathered}
u_{1}=1, \quad \tilde{u}_{1}=\frac{\Omega_{12} c_{x}^{2}+\Omega_{21} c_{y}^{2}}{2\left(\Omega_{12}+\Omega_{21}\right)}, \\
u_{2}=\frac{2\left(\Omega_{21} c_{x}^{2}-\Omega_{12} c_{y}^{2}\right)}{\Omega_{12}+\Omega_{21}}, \quad \tilde{u}_{2}=\frac{1}{4}\left(c_{x}^{2}-c_{y}^{2}\right), \\
u_{3}=c_{x}, \quad \tilde{u}_{3}=\frac{1}{2} c_{x}, \\
u_{4}=c_{y}, \quad \tilde{u}_{4}=\frac{1}{2} c_{y},
\end{gathered}
$$

with eigenvalues given by

$$
\begin{gathered}
\omega_{1}=0, \quad \omega_{2}=2\left(\Omega_{12}+\Omega_{21}\right), \\
\omega_{3}=\Omega_{13}-\Omega_{11}, \quad \omega_{4}=\Omega_{24}-\Omega_{22} .
\end{gathered}
$$

The asymmetry $\Omega_{12} \neq \Omega_{21}$ leads to the mixing between the vectors 1 and $c_{x}^{2}-c_{y}^{2}$. If the model is symmetric for interchange between the $x$ and $y$ directions, then $\Omega_{21}=\Omega_{12}$, $\Omega_{22}=\Omega_{11}$, and $\Omega_{24}=\Omega_{13}$.

This is the relevant set of eigenfunctions for the asymmetric interactions of Sec. III. In Sec. IV the interactions do not break the symmetry between the $x$ and $y$ directions, and the eigenfunctions and eigenvalues simplify. A table similar to Table I which includes the behavior under the symmetry $x \leftrightarrow y$ can be constructed for this case. All four vectors 1 , $c_{x}^{2}-c_{y}^{2}, c_{x}$, and $c_{y}$ now span one-dimensional invariant subspaces; so that the eigenvectors for the symmetric case are

$$
\begin{gathered}
u_{1}=1, \quad \tilde{u}_{1}=\frac{1}{4} u_{1}, \\
u_{2}=c_{x}^{2}-c_{y}^{2}, \quad \widetilde{u_{2}}=\frac{1}{4} u_{2}, \\
u_{3}=c_{x}, \quad \widetilde{u_{3}}=\frac{1}{2} u_{3}, \\
u_{4}=c_{y}, \quad \widetilde{u_{4}}=\frac{1}{2} u_{4} .
\end{gathered}
$$

The corresponding eigenvalues are given by

$$
\omega_{1}=0, \quad \omega_{2}=4 \Omega_{12}, \quad \omega_{3}=\omega_{4}=2\left(\Omega_{12}+\Omega_{13}\right) .
$$

\section{Structure of $E$}

The eigenvalues $\epsilon_{n}$ of the symmetric source matrix $E$, defined in Eqs. (9) and (12), are defined by

$$
E v_{n}=\epsilon_{n} v_{n} .
$$

In the asymmetric case of Sec. III, the inversion symmetry in the $x$ and $y$ axes together with the symmetry $E_{j i}=E_{i j}$ imposes the structure

$$
E=\left(\begin{array}{llll}
E_{11} & E_{12} & E_{13} & E_{12} \\
E_{12} & E_{22} & E_{12} & E_{24} \\
E_{13} & E_{12} & E_{11} & E_{12} \\
E_{12} & E_{24} & E_{12} & E_{22}
\end{array}\right) .
$$

The conservation laws $\langle 1|E| 1\rangle=0$ [see Eq. (14)] imposes one more relation between the matrix elements $E_{i j}$. Because of the symmetry $E=E^{T}$ there is no distinction between left and right eigenvectors. The symmetry argument of Table I of course also holds for $E$. We therefore know that

$$
v_{3}=c_{x}, \quad v_{4}=c_{y},
$$

are two eigenvectors with eigenvalues

$$
\epsilon_{3}=E_{11}-E_{13}, \quad \epsilon_{4}=E_{22}-E_{24} .
$$

The two remaining eigenvalues are the solutions of the quadratic equation

$$
\begin{array}{cl}
\epsilon^{2}- & \epsilon\left(E_{11}+E_{22}+E_{13}+E_{24}\right)+\left(E_{13}+E_{11}\right)\left(E_{12}+E_{24}\right)-4 E_{12}^{2} \\
\quad=0, & \text { (A13) }
\end{array}
$$

and the corresponding eigenvectors are linear combinations of $c_{x}^{2}$ and $c_{y}^{2}$. Their explicit form will not be needed in the present paper.

In the symmetric case of Sec. IV, when there is no difference between $x$ and $y$ directions, we find that $\Omega$ and $E$ have the same set of eigenvectors,

$$
v_{1}=1, \quad v_{2}=c_{x}^{2}-c_{y}^{2}, \quad v_{3}=c_{x}, \quad v_{4}=c_{y},
$$

with eigenvalues

$$
\epsilon_{1}=0, \quad \epsilon_{2}=-4 E_{12}, \quad \epsilon_{3}=\epsilon_{4}=E_{11}-E_{13} .
$$

Here $\epsilon_{1}=0$ follows from the conservation law $\langle 1|E| 1\rangle=0$ together with the symmetry properties of $E$.

\section{Asymmetric interacting random walkers}

Inspection of Sec. III shows that we need to calculate the diffusion coefficients $D_{x}$ and $D_{y}$ in Eq. (41) and the projections in Eq. (43), which also defines the source terms $B_{x}$ and $B_{y}$. To determine these quantities we have to calculate

$$
\psi_{D}(\mathbf{q}, \mathbf{c})=\psi_{0}+(i q) \psi_{1}+(i q)^{2} \psi_{2} .
$$

The eigenvalue equations (23) show the symmetry properties

$$
\begin{gathered}
\psi_{D}(\mathbf{q}, \mathbf{c})=\psi_{D}(-\mathbf{q},-\mathbf{c})=\psi_{+}(\mathbf{q}, \mathbf{c})+\psi_{-}(\mathbf{q}, \mathbf{c}), \\
z_{D}(\mathbf{q})=z_{D}(-\mathbf{q}) \simeq(i q)^{2} D+\cdots,
\end{gathered}
$$


where $\psi_{ \pm}$has an even $(+)$or odd $(-)$parity in $\mathbf{q}$ and $\mathbf{c}$ separately. With the help of these equations we easily obtain

$$
\begin{aligned}
B_{x} \hat{q}_{x}^{2}+B_{y} \hat{q}_{y}^{2} & =\frac{1}{2}\left\langle\psi_{D}(\mathbf{q})|E| \psi_{D}(-\mathbf{q})\right\rangle \\
& =\frac{1}{2}\left\langle\psi_{1}|E| \psi_{1}\right\rangle-\left\langle\psi_{0}|E| \psi_{2}\right\rangle .
\end{aligned}
$$

Here the relations $\psi_{0}=1$ and $\langle 1|E| 1\rangle=0$ have been used.

The solution of Eq. (28a) is $\psi_{0}=1=u_{1}$, and similarly $\widetilde{\psi}_{0}=\widetilde{u_{1}}$, where $u_{n}$ and $\widetilde{u_{n}}$ are defined in Appendix A 1 . We choose the normalization of $\psi_{D}(\mathbf{q})$ such that its component parallel to $u_{1}$ is unity to all orders in the perturbation. This implies

$$
\begin{aligned}
& \left\langle\tilde{u}_{1} \mid \psi_{D}\right\rangle=\left\langle\widetilde{u_{1}} \mid \psi_{0}\right\rangle=1, \\
& \left\langle\tilde{u}_{1} \mid \psi_{n}\right\rangle=0 \quad(n \geqslant 1) .
\end{aligned}
$$

The solution of the second order equation (28b), where the inhomogeneous term $I_{D}^{(1)}$ is a linear combination of $u_{3}$ and $u_{4}$, then becomes

$$
\psi_{1}=\frac{1}{\Omega^{T}} c_{\ell}=-\left(\frac{\hat{q}_{x} c_{x}}{\omega_{3}}+\frac{\hat{q}_{y} c_{y}}{\omega_{4}}\right) .
$$

Before we can determine $\psi_{2}$ we impose the solubility conditions

$$
\left\langle\widetilde{u}_{1} \mid I_{D}^{(2)}\right\rangle=\left\langle\widetilde{u}_{1} c_{\ell} \mid \psi_{1}\right\rangle+\left\langle\widetilde{u_{1}} \mid D(\hat{\mathbf{q}})+\frac{1}{2} c_{\ell}^{2}\right\rangle .
$$

We obtain in a straightforward manner

$$
D(\hat{\mathbf{q}})=D_{x} \hat{q}_{x}^{2}+D_{y} \hat{q}_{y}^{2}=-\left\langle\tilde{u_{1}} c_{\ell}\left|\frac{1}{\Omega^{T}}+\frac{1}{2}\right| c_{\ell}\right\rangle .
$$

Working this out yields

$$
\begin{aligned}
& D_{x}=\frac{\Omega_{12}}{\Omega_{12}+\Omega_{21}}\left(\frac{1}{\omega_{3}}-\frac{1}{2}\right), \\
& D_{y}=\frac{\Omega_{21}}{\Omega_{12}+\Omega_{21}}\left(\frac{1}{\omega_{4}}-\frac{1}{2}\right) .
\end{aligned}
$$

The eigenfunction can be solved from Eq. (28c) as

$$
\psi_{2}=\frac{1}{4}\left(\frac{D_{x} \hat{q}_{x}^{2}}{\Omega_{12}}-\frac{D_{y} \hat{q}_{y}^{2}}{\Omega_{21}}\right) u_{2}
$$

Inserting the results of Eqs. (A20) and (A24) into Eq. (A18) and using the properties of the $E$ matrix in Appendix A 2 allows us to obtain the coefficients $B_{\alpha}$ in Eq. (A18) as

$$
\begin{aligned}
& B_{x}=\frac{\epsilon_{3}}{\omega_{3}^{2}}-\frac{D_{x}}{2 \Omega_{12}}\left(E_{11}+E_{13}-E_{22}-E_{24}\right), \\
& B_{y}=\frac{\epsilon_{4}}{\omega_{4}^{2}}+\frac{D_{y}}{2 \Omega_{21}}\left(E_{11}+E_{13}-E_{22}-E_{24}\right) .
\end{aligned}
$$

In deriving this result we have used the relations $\langle 1|E| 1\rangle=0$ and

$$
\left\langle 1|E| u_{2}\right\rangle=2\left(E_{11}+E_{13}-E_{22}-E_{24}\right) .
$$

The projection in Eq. (43) simply yields

$$
\left\langle 11 \mid \widetilde{\psi}_{D}(\mathbf{q}) \widetilde{\psi}_{D}(-\mathbf{q})\right\rangle=\left[\left\langle 1 \mid \widetilde{\psi}_{0}\right\rangle\right]^{2}=1,
$$

because of the normalization (A19).

\section{Symmetric interacting random walkers}

The required calculations for Sec. IV are much more involved, as the eigenvalue $z_{D}(\mathbf{q})$ in Eq. (48) and the source terms in Eq. (50) must be evaluated up to terms $O\left(q^{4}\right)$. This requires the perturbation equations for $\psi_{0}, \psi_{1}, \ldots, \psi_{4}$. However, a substantial simplification occurs because the linearized Boltzmann collision operator is now symmetric, $\Omega=\Omega^{T}$, and right and left eigenfunctions are the same.

The calculations are tedious but straightforward, and we quote some intermediate as well as final results. The coefficients of the diffusion mode $\psi_{D}(\mathbf{q})=\sum_{n=0}^{4}(i q)^{n} \psi_{n}$ are

$$
\begin{gathered}
\psi_{0}=1, \quad \psi_{1}=-\frac{1}{\omega_{3}} c_{\ell}, \\
\psi_{2}=\frac{2 D}{\omega_{2}}\left(c_{\ell}^{2}-\frac{1}{2}\right),
\end{gathered}
$$

$$
\psi_{3}=-\frac{1}{\omega_{3}}\left(4 D \Theta-\frac{1}{12}\right) c_{\ell}^{3}+\frac{2 D}{\omega_{3}}(D+\Theta) c_{\ell} .
$$

Here the transport coefficients are

$$
D=\frac{1}{2}\left(\frac{1}{\omega_{3}}-\frac{1}{2}\right), \quad \Theta=\frac{1}{2}\left(\frac{1}{\omega_{2}}-\frac{1}{2}\right),
$$

and the super-Burnett coefficient is found as

$$
D_{2}(\hat{\mathbf{q}})=D_{2}^{\prime}\left(\hat{q}_{x}^{4}+\hat{q}_{y}^{4}\right)+D_{2}^{\prime \prime}=-2 D_{2}^{\prime} \hat{q}_{x}^{2} \hat{q}_{y}^{2}+D_{2}^{\prime \prime \prime} .
$$

It has an anisotropic part $D_{2}^{\prime}$ and an isotropic part, whose explicit form is not needed in this paper. Only the former part enters into the coefficient [see Eqs. (50) and (51)] of the algebraic tail $\sim 1 / r^{4}$ of the pair correlation function, and it reads

$$
D_{2}^{\prime}=4 D\left(D \Theta-\frac{1}{24}\right) .
$$

The source terms in Eq. (50) are determined by

$$
B=\frac{1}{2}\left\langle\psi_{1}|E| \psi_{1}\right\rangle, \quad B_{2}(\hat{\mathbf{q}})=\frac{1}{2}\left\langle\psi_{2}|E| \psi_{2}\right\rangle-\left\langle\psi_{1}|E| \psi_{3}\right\rangle,
$$

and the results (A28) enable us to calculate these contributions as

$$
\begin{gathered}
B=\frac{1}{2 \omega_{3}^{2}}\left\langle c_{\ell}|E| c_{\ell}\right\rangle=\frac{\epsilon_{3}}{\omega_{3}^{2}}, \\
B_{2}(\hat{\mathbf{q}})=B_{2}^{\prime}\left(\hat{q}_{x}^{4}+\hat{q}_{y}^{4}\right)+B_{2}^{\prime \prime}=-2 B_{2}^{\prime} \hat{q}_{x}^{2} \hat{q}_{y}^{2}+B_{2}^{\prime \prime \prime} .
\end{gathered}
$$

Again, only the anisotropic part enters the amplitude of the algebraic tail, and is given by 


$$
B_{2}^{\prime}=\frac{4 \epsilon_{2} D^{2}}{\omega_{2}^{2}}+\frac{\epsilon_{3}}{\omega_{3}^{2}}\left(8 D \Theta-\frac{1}{6}\right)
$$

Finally, Eq. (A27) holds for the symmetric case as well.

\section{APPENDIX B: FLUID-TYPE MODEL}

The goal of this appendix is to calculate the left and right hydrodynamic modes $\left[\psi_{\mu}(\mathbf{q})\right.$ and $\widetilde{\psi}_{\mu}(\mathbf{q})$ for small wave number $q$ ] of the lattice Boltzmann equation (23) for a fluidtype LGA, defined on a triangular lattice, that conserves both particle number and momentum during collisions. More specifically, we need to determine the expansion coefficients $\psi_{\mu}^{(n)}(n=0,1,2)$ and $\widetilde{\psi}_{\mu}^{(n)}(n=0)$ of these modes in powers of $i q$, as well as the coefficients $\left\langle\psi_{\mu}^{(n)}|E| \psi_{\mu}^{(m)}\right\rangle$.

A basic ingredient in this calculation is the structure of the linearized Boltzmann collision operator $\Omega_{i j}(i=0,1, \ldots, 6)$ in Eq. (13), which is a nonsymmetric matrix because the LGA under consideration violates the semidetailed-balance condition (4). The appendix is organized as follows. In Sec. B 1 the eigenvectors and eigenvalues of $\Omega$ are calculated in terms of its matrix elements, using the triangular symmetry of the lattice; in Sec. B 2 the same is done for the symmetric source matrix defined in Eqs. (9) and (12). Section B 3 calculates the coefficients $\psi_{\mu}^{(n)}$ and $\left\langle\psi_{\mu}^{(n)}|E| \psi_{\mu}^{(m)}\right\rangle$ in so far as they are needed in the body of the paper.

\section{Structure of $\Omega$}

The left and right eigenvectors $u_{n}$ and $\widetilde{u}_{n}$ and the corresponding eigenvalues $-\omega_{n}$ of the nonsymmetric $\Omega$ are defined as

$$
\Omega^{T} u_{n}=-\omega_{n} u_{n}, \quad \Omega \widetilde{u_{n}}=-\omega_{n} \tilde{u_{n}},
$$

where $\left(\Omega^{T}\right)_{i j}=\Omega_{j i}$ and $i, j=0,1, \ldots, 6$. The left and right eigenvectors together form a biorthogonal set, normalized as $\left\langle u_{n} \mid \widetilde{u}_{m}\right\rangle=\delta_{n m}$. The lattice symmetries of the triangular lattice impose the general structure

$$
\Omega=\left(\begin{array}{ccccccc}
\alpha_{0} & \alpha_{1} & \alpha_{1} & \alpha_{1} & \alpha_{1} & \alpha_{1} & \alpha_{1} \\
\widetilde{\alpha}_{1} & \alpha & \beta & \gamma & \delta & \gamma & \beta \\
\widetilde{\alpha_{1}} & \beta & \alpha & \beta & \gamma & \delta & \gamma \\
\widetilde{\alpha}_{1} & \gamma & \beta & \alpha & \beta & \gamma & \delta \\
\widetilde{\alpha}_{1} & \delta & \gamma & \beta & \alpha & \beta & \gamma \\
\widetilde{\alpha}_{1} & \gamma & \delta & \gamma & \beta & \alpha & \beta \\
\widetilde{\alpha}_{1} & \beta & \gamma & \delta & \gamma & \beta & \alpha
\end{array}\right)
$$

where the submatrix $\left\{\Omega_{i j} ; i, j=1, \ldots, 6\right\}$ is symmetric. We frequently use a notation where a seven-vector $v(\mathbf{c})$ with components $v_{i}(\mathbf{c})=v\left(\mathbf{c}_{i}\right)(i=0,1, \ldots, b)$ will be denoted as $\left(v\left(\mathbf{c}_{0}\right) \mid v(\mathbf{c})\right)$ or $\left(v\left(\mathbf{c}_{0}\right) \mid v\left(\mathbf{c}_{i}\right)\right)$. The first component $v\left(\mathbf{c}_{0}\right)$ refers to the rest particle state with $\mathbf{c}_{0}=\mathbf{0}$, and the remaining components $(i=1,2, \ldots, 6)$ refer to moving particle states. The conservation law (14) implies that the set of collisional invariants

$$
a_{\alpha}=\left\{a_{\rho}, a_{\ell}, a_{\perp}\right\}=\left\{1, c_{\ell}, c_{\perp}\right\}=\left\{u_{0}, u_{2}, u_{3}\right\}
$$

TABLE II. Left and right eigenvectors $u_{n i}=u_{n}\left(\mathbf{c}_{i}\right)$ with $i=0,1, \ldots, b$, and eigenvalues $-\omega_{n}$ of the linearized Boltzmann operator $\Omega$ for a seven-bit fluid-type LGA on a triangular lattice.

\begin{tabular}{cccc}
\hline \hline$n$ & $u_{n}(\mathbf{c})$ & $\widetilde{u}_{n}(\mathbf{c})$ & $-\omega_{n}$ \\
\hline 0 & $a_{\rho}=1$ & $\widetilde{a}_{\rho}=\left(1-2 v_{s}^{2} \mid \frac{1}{3} v_{s}^{2}\right)$ & 0 \\
1 & $\frac{1}{2} c^{2}-v_{s}^{2}$ & $\left(-2 \mid \frac{1}{3}\right)$ & $-\omega_{1}$ \\
2 & $a_{\ell}=c_{\ell}$ & $\widetilde{a}_{\ell}=\frac{1}{3} u_{2}$ & 0 \\
3 & $a_{\perp}=c_{\perp}$ & $\widetilde{a}_{\perp}=\frac{1}{3} u_{3}$ & 0 \\
4 & $c, c_{\perp}$ & $\frac{4}{3} u_{4}$ & $-\omega_{4}$ \\
5 & $c_{\ell}^{2}-\frac{1}{2} c^{2}$ & $\frac{4}{3} u_{5}$ & $-\omega_{5}$ \\
6 & $\left(0 \mid(-1)^{i+1}\right)$ & $\frac{1}{6} u_{6}$ & $-\omega_{6}$ \\
\hline \hline
\end{tabular}

(see Table II) are left eigenfunctions, i.e., $\Omega^{T} a_{\alpha}=0$. Multiplication of the matrix (B2) on the left with $u_{0}=(1 \mid 1)$ and $u_{2}=\left(0 \mid c_{\ell}\right)$ imposes the conditions

$$
\begin{gathered}
\alpha_{0}+6 \widetilde{\alpha_{1}}=0, \\
\alpha_{1}+\alpha+2 \beta+2 \gamma+\delta=0, \\
\alpha+\beta-\gamma-\delta=0 .
\end{gathered}
$$

Because of the symmetry $\Omega_{i j}=\Omega_{j i}$ for $i, j=1,2, \ldots, 6$ the eigenvectors $u_{n}(n=2,3)$ are proportional to right zero eigenvectors $\widetilde{u_{2}}=\tilde{a}_{\ell}$ and $\tilde{u}_{3}=\tilde{a}_{\perp}$ (see Table II). To construct the right zero eigenvector $\widetilde{u_{0}}$ we note that by symmetry it must have the structure $\widetilde{u_{0}}=\left(x_{0} \mid x\right)$, and satisfy $\Omega \widetilde{u_{0}}=0$ with $\left\langle u_{0} \mid \widetilde{u}_{0}\right\rangle=1$. The result is

$$
\widetilde{u_{0}} \equiv \widetilde{a_{\rho}}=\frac{1}{\alpha_{1}+6 \widetilde{\alpha_{1}}}\left(\alpha_{1} \mid \widetilde{\alpha_{1}}\right)=\frac{1}{\alpha_{1}-\alpha_{0}}\left(\alpha_{1} \mid-\frac{1}{6} \alpha_{0}\right) .
$$

It is possible to identify the components of $\widetilde{u_{0}}$ in terms of the equilibrium distribution function $f_{i}(\rho)=\left(f_{0}(\rho) \mid f(\rho)\right)$, which is the stationary solution of the nonlinear Boltzmann equation (7), $\Omega_{i}^{1,0}(f(\rho))=0$, at a given density $\rho=f_{0}+6 f$ [16]. Then we have for an infinitesimal change $d \rho$ in the density

$$
0=\Omega_{i}^{1,0}(f(\rho+d \rho))=\sum_{j} \Omega_{i j}(f(\rho)) \frac{d f_{j}}{d \rho} d \rho,
$$

where we have used the definition of $\Omega_{i j}$ given above in Eq. (13). This allows us to identify

$$
\widetilde{u_{0}}=\left(\frac{d f_{0}}{d \rho} \mid \frac{d f}{d \rho}\right)=\left(1-2 v_{s}^{2} \mid \frac{1}{3} v_{s}^{2}\right),
$$

where the speed of sound $v_{s}$ is defined by

$$
v_{s}^{2}=\frac{d p}{d \rho}=3 \frac{d f}{d \rho}=\frac{1}{2}\left(1-\frac{d f_{0}}{d \rho}\right),
$$

with $p=\Sigma_{i} c_{/ i}^{2} f_{i}=3 f=\frac{1}{2}\left(\rho-f_{0}\right)$. One also verifies from Eq. (B7) that $\left\langle u_{0} \mid \widetilde{u_{0}}\right\rangle=1$. From the identification of Eqs. (B5) and (B7) we obtain

$$
v_{s}^{2}=\frac{1}{2}\left(\frac{\alpha_{0}}{\alpha_{0}-\alpha_{1}}\right)=\frac{1}{2}\left(\frac{\Omega_{00}}{\Omega_{00}-\Omega_{01}}\right) .
$$


TABLE III. Eigenvectors $v_{n}(\mathbf{c})$ and eigenvalues $\epsilon_{n}$ of the source matrix $E$ for a seven-bit fluid-type LGA on a triangular lattice.

\begin{tabular}{cccc}
\hline \hline$n$ & $v_{n}$ & $\widetilde{v_{n}}$ & $\epsilon_{n}$ \\
\hline 0 & linear comb. & linear comb. & $\epsilon_{0} \neq 0$ \\
1 & of $u_{0}$ and $u_{1}$ & of $\widetilde{u_{0}}$ and $\widetilde{u_{1}}$ & $\epsilon_{1} \neq 0$ \\
2 & $u_{2}$ & $\widetilde{u_{2}}$ & 0 \\
3 & $u_{3}$ & $\widetilde{u_{3}}$ & 0 \\
4 & $u_{4}$ & $\widetilde{u_{4}}$ & $\epsilon_{4}$ \\
5 & $u_{5}$ & $\widetilde{u_{5}}$ & $\epsilon_{5}$ \\
6 & $u_{6}$ & $\widetilde{u_{6}}$ & $\epsilon_{6}$ \\
\hline \hline
\end{tabular}

Table II shows the complete set of right and left eigenvectors of $\Omega$. Most eigenvectors can be found on the basis of symmetry arguments alone, except $u_{1}$ and $\tilde{u}_{1}$. The corresponding eigenvalues are found as

$$
\begin{gathered}
\omega_{1}=\Omega_{10}-\Omega_{00}, \quad \omega_{4}=\omega_{5}=2\left(\Omega_{12}-\Omega_{14}\right), \\
\omega_{6}=3\left(\Omega_{12}-\Omega_{13}\right) .
\end{gathered}
$$

\section{Structure of $E$}

So far we have calculated the eigenvectors and eigenvalues of $\Omega$. In a similar manner we can do so for the source matrix $E_{i j}$ defined in Eq. (9) or (12). Then

$$
E v_{n}=\epsilon_{n} v_{n} .
$$

As $E_{i j}=E_{j i}$ is symmetric, left and right eigenvectors are the same up to a normalization factor, i.e., $\widetilde{v_{n}}=v_{n} /\left\langle v_{n} \mid v_{n}\right\rangle$. The structure of $E$ is also given by Eq. (B2), with $\widetilde{\alpha}_{1}=\alpha_{1}$. The lattice symmetry of the submatrix $\left\{E_{i j} ; i, j=1,2, \ldots, 6\right\}$ implies that $v_{n}=u_{n}$ for $n=2,3, \ldots, 6$. The conservation laws (14) imply

$$
\langle 1|E| 1\rangle=\left\langle c_{\ell}|E| c_{\ell}\right\rangle=\left\langle c_{\perp}|E| c_{\perp}\right\rangle=0,
$$

which imposes two relations between the matrix elements $E_{i j}$, and implies that $v_{2}=c_{\ell}$ and $v_{3}=c_{\perp}$ are zero eigenvectors with $\epsilon_{2}=\epsilon_{3}=0$. However, $\epsilon_{0} \neq 0$, and the corresponding eigenvector $v_{0}$ is a linear combination of $u_{0}$ and $u_{1}$. The remaining eigenvalues in terms of $E_{i j}$ are obtained from Eqs. (B10) and (B12) and read

$$
\begin{gathered}
\epsilon_{4}=\epsilon_{5}=2\left(E_{14}-E_{12}\right)=2\left(E_{11}-E_{13}\right), \\
\epsilon_{6}=3\left(E_{13}-E_{12}\right)=3\left(E_{11}-E_{14}\right) .
\end{gathered}
$$

The results are summarized in Table III.

\section{Perturbation theory to $O\left(q^{2}\right)$}

In this appendix we calculate the hydrodynamic modes and matrix elements occurring in Eqs. (58) and (62) by means of perturbation theory for degenerate eigenvalues. We use as a basis the eigenvectors $u_{n}$ and $\tilde{u}_{n}$ of $\Omega$ that were constructed in Sec. B 1. Our starting point is the observation that according to Eq. (28a) a hydrodynamic zeroth order mode $\psi_{\mu}^{(0)}$ will be a linear combination of collisional invariants:

$$
\psi_{\mu}^{(0)}=C_{\mu \rho} a_{\rho}+C_{\mu \ell} a_{\ell}+C_{\mu \perp} a_{\perp}=\sum_{\beta} C_{\mu \beta} a_{\beta} .
$$

The solubility condition (30) for $\psi_{\mu}^{(0)}$ yields the equation $\Sigma_{\beta}\left\langle\tilde{a}_{\alpha}\left|c_{\ell}+z_{\mu}^{(1)}\right| a_{\beta}\right\rangle C_{\mu \beta}=0$ with $\alpha=\rho, \ell, \perp$. Nonzero solutions $C_{\mu \alpha}$ only exist if $z_{\mu}^{(1)}$ satisfies the secular equation,

$$
\operatorname{det}\left|\left\langle\tilde{a}_{\alpha}\left|c_{\ell}+z_{\mu}^{(1)}\right| a_{\beta}\right\rangle\right|=0 \text {. }
$$

The only nonvanishing elements are $\left\langle\tilde{a}_{\rho}\left|c_{\ell}\right| a_{\ell}\right\rangle=v_{s}^{2}$ and $\left\langle\tilde{a}_{\ell}\left|c_{\ell}\right| a_{\rho}\right\rangle=1$, and furthermore $\left\langle\tilde{a}_{\alpha} \mid a_{\beta}\right\rangle=\delta_{\alpha \beta}$. From the secular equation we find three nondegenerate eigenvalues $z_{\mu}^{(1)}$, and subsequently we can determine the coefficients $C_{\mu \alpha}$ up to a normalization constant. Thus the threefold degeneracy of the null space of $\Omega$ is lifted, and we have a right shear mode $\mu=\perp$,

$$
\psi_{\perp}^{(0)}=c_{\perp}, \quad z_{\perp}^{(1)}=0
$$

and a pair of right sound modes $\mu=\sigma= \pm$,

$$
\psi_{\sigma}^{(0)}=a_{\ell}+\sigma v_{s} a_{\rho}, \quad z_{\sigma}^{(1)}=-\sigma v_{s} .
$$

In a similar manner we obtain the corresponding left eigenvectors of Eq. (B15) as

$$
\begin{gathered}
\widetilde{\psi}_{\perp}^{(0)}=\widetilde{a}_{\perp}=\frac{1}{3} c_{\perp}, \\
\widetilde{\psi}_{\sigma}^{(0)}=\frac{1}{2}\left(\widetilde{a}_{\ell}+\frac{\sigma}{v_{s}} \widetilde{a}_{\rho}\right) .
\end{gathered}
$$

Next we consider the first order left hydrodynamic modes. The right modes $\widetilde{\psi}_{\mu}^{(n)}$ are only required to zeroth order $(n=0)$. The formal solution of Eq. (28b) is

$$
\psi_{\mu}^{(1)}=\frac{1}{\Omega^{T}}\left(c_{\ell}+z_{\mu}^{(1)}\right) \psi_{\mu}^{(0)}+\sum_{\lambda} B_{\mu \lambda}^{(1)} \psi_{\lambda}^{(0)}
$$

It contains an arbitrary linear combination of zeroth order modes. We always choose the normalization such that the projection of $\psi_{\mu}$ onto $\psi_{\mu}^{(0)}$ is unity, i.e., $\left\langle\widetilde{\psi}_{\mu}^{(0)} \mid \psi_{\mu}\right\rangle=\left\langle\widetilde{\psi}_{\mu}^{(0)} \mid \psi_{\mu}^{(0)}\right\rangle=1$ and consequently for $n \geqslant 1$

$$
\left\langle\widetilde{\psi}_{\mu}^{(0)} \mid \psi_{\mu}^{(n)}\right\rangle=0 \text { or } B_{\mu \mu}^{(n)}=0 .
$$

The corresponding coefficients $\widetilde{B}_{\mu \mu}^{(n)}$ in the right eigenmodes $\widetilde{\psi}_{\mu}^{(n)}$ are then determined by the normalization conditions (29). They are, however, not needed in the present paper. The remaining coefficients $B_{\mu \lambda}^{(1)}(\lambda \neq \mu)$ as well as the next order eigenvalue $z_{\mu}^{(2)}$ are determined from the solubility conditions of the second order perturbation equations (30). The method to determine $B_{\mu \lambda}^{(1)}$ has been explained in Ref. [17] for a symmetric collision operator $\Omega$, but the steps are all very similar. In this manner we find for the eigenvalues to relevant order 


$$
\begin{gathered}
z_{\perp}(\mathbf{q})=-\nu q^{2}, \\
z_{\sigma}(\mathbf{q})=-i q \sigma v_{s}-\Gamma q^{2},
\end{gathered}
$$

where $v_{s}$ is the speed of sound, given in Eq. (B9), while $\Gamma=\frac{1}{2}(\nu+\zeta)$ is the sound damping constant, with $\nu$ and $\zeta$ the shear and bulk viscosity, respectively, given in terms of the eigenvalues $-\omega_{n}$ as (see Table II)

$$
\nu=\frac{1}{4}\left(\frac{1}{\omega_{4}}-\frac{1}{2}\right), \quad \zeta=\left(\frac{1}{2}-v_{s}^{2}\right)\left(\frac{1}{\omega_{1}}-\frac{1}{2}\right) .
$$

The eigenmodes to first order are found as

$$
\begin{gathered}
\psi_{\perp}^{(1)}=\frac{1}{\Omega^{T}} c c_{\perp}=-\frac{1}{\omega_{4}} u_{4}, \\
\psi_{\sigma}^{(1)}=\frac{1}{\Omega^{T}}\left(c_{\ell}-\sigma v_{s}\right) \psi_{\sigma}^{(0)}+B_{\sigma,-\sigma} \psi_{-\sigma}^{(0)} \\
=\frac{1}{\Omega^{T}}\left(c_{\ell}^{2}-v_{s}^{2}\right)-\frac{\sigma \Gamma}{2 v_{s}}\left(c_{\ell}-\sigma v_{s}\right) \\
=-\frac{u_{5}}{\omega_{5}}-\frac{u_{1}}{\omega_{1}}+\frac{\Gamma}{2} u_{0}-\frac{\sigma \Gamma}{2 v_{s}} u_{2},
\end{gathered}
$$

where Table II has been used. The coefficients $B_{\perp \lambda}=0$ and $B_{\sigma \lambda}=0$ for $\lambda \neq \sigma$.

The results so far are sufficient to calculate the transverse susceptibility using Eq. (59). From Table III we conclude that $E \psi_{\perp}^{(0)}=E u_{3}=0$, and consequently $\left\langle\psi_{\perp}^{(0)}|E| \psi_{\perp}^{(2)}\right\rangle=0$. The remaining term in Eq. (59), combined with Eq. (B23) then yields

$$
\Delta \chi_{\perp}(\mathbf{q})=\frac{\epsilon_{4}}{2 \nu \omega_{4}^{2}}\left\langle u_{4} \mid u_{4}\right\rangle=\frac{3 \epsilon_{4}}{8 \nu \omega_{4}^{2}},
$$

where $E u_{4}=\epsilon_{4} u_{4}$ (see Table III).

Next we consider the contributions entering the longitudinal susceptibility in Eq. (62), starting with the parallel sound modes. To calculate the matrix elements in Eq. (63) we observe that $E \psi_{\sigma}^{(0)}=\sigma v_{s} E u_{0}$ (see Table III). This permits us to combine the terms on the right hand side of Eq. (63) into

$$
\begin{aligned}
\left\langle\psi_{\sigma}(\mathbf{q})|E| \psi_{-\sigma}^{*}(\mathbf{q})\right\rangle & =-i q \sigma v_{s}\left\langle u_{0}|E| \psi_{\sigma}^{(1)}+\psi_{-\sigma}^{(1)}\right\rangle \\
& =2 i q \sigma v_{s}\left[\frac{1}{\omega_{5}} \mathcal{E}_{05}+\frac{1}{\omega_{1}} \mathcal{E}_{01}-\frac{1}{2} \Gamma \mathcal{E}_{00}\right] .
\end{aligned}
$$

To obtain the second line we have inserted Eq. (B23) and introduced

$$
\mathcal{E}_{n m}=\left\langle u_{n}|E| u_{m}\right\rangle .
$$

According to Table III, $u_{5}=v_{5}$ is an eigenvector of $E$, orthogonal to the subspace spanned by $u_{0}$ and $u_{1}$; consequently $\mathcal{E}_{05}=0$. Moreover, $\mathcal{E}_{00}=\langle 11 \mid E\rangle=0$ because of the conservation laws (14). This yields the result listed in Eq. (63). To calculate the contributions of the opposite sound modes we need $\left\langle\psi_{\sigma}^{(0)}|E| \psi_{\sigma}^{(2)}\right\rangle$ in Eq. (64). Using $E \psi_{\sigma}^{(0)}=\sigma v_{s} E u_{0}$ we write

$$
\begin{aligned}
\left\langle\psi_{\sigma}^{(0)}|E| \psi_{\sigma}^{(2)}\right\rangle & =\sigma v_{s}\left\langle u_{0}|E|\left\{u_{0}\left\langle\tilde{u}_{0} \mid \psi_{\sigma}^{(2)}\right\rangle+u_{1}\left\langle\tilde{u}_{1} \mid \psi_{\sigma}^{(2)}\right\rangle\right\}\right\rangle \\
& =\sigma v_{s} \mathcal{E}_{01}\left\langle\widetilde{u}_{1} \mid \psi_{\sigma}^{(2)}\right\rangle .
\end{aligned}
$$

To obtain the first equality we note that $\mathcal{E}_{00}=0$ and that all off-diagonal elements $\mathcal{E}_{n m}=0(n \neq m)$, except $\mathcal{E}_{01} \neq 0$ (see Table III).

The coefficient $\left\langle\widetilde{u}_{1} \mid \psi_{\sigma}^{(2)}\right\rangle$ in Eq. (B26) can be calculated most conveniently by projecting the second order eigenvalue equation (28c) onto the vector $\widetilde{u_{1}}$, i.e.,

$$
\begin{aligned}
\left\langle\widetilde{u}_{1} \mid \Omega^{T} \psi_{\sigma}^{(2)}\right\rangle= & \left\langle\widetilde{u}_{1}\left(c_{\ell}-\sigma v_{s}\right) \mid \psi_{\sigma}^{(1)}\right\rangle+\Gamma\left\langle\widetilde{u}_{1} \mid \psi_{\sigma}^{(0)}\right\rangle \\
& +\frac{1}{2}\left\langle\widetilde{u_{1}}\left(c_{\ell}-\sigma v_{s}\right) \mid\left(c_{\ell}^{2}-v_{s}^{2}\right)\right\rangle .
\end{aligned}
$$

Substituting Eq. (B23) and using the relations

$$
\begin{gathered}
\tilde{u}_{1}\left(c_{\ell}-\sigma v_{s}\right)=\tilde{u_{2}}-\sigma v_{s} \tilde{u}_{1}, \\
c_{\ell}^{2}-v_{s}^{2}=u_{5}+u_{1}, \\
\left\langle\widetilde{u}_{1} \mid \psi_{\sigma}^{(0)}\right\rangle=0, \\
\left\langle\widetilde{u}_{1} \mid \Omega^{T} \psi_{\sigma}^{(2)}\right\rangle=-\omega_{1}\left\langle\widetilde{u}_{1} \mid \psi_{\sigma}^{(2)}\right\rangle,
\end{gathered}
$$

we find for the coefficient

$$
\left\langle\widetilde{u}_{1} \mid \psi_{\sigma}^{(2)}\right\rangle=\frac{\sigma}{2 v_{s} \omega_{1}}\left[\Gamma+v_{s}^{2}-\frac{2 v_{s}^{2}}{\omega_{1}}\right] .
$$

The first term in Eq. (64), $\left\langle\psi_{\sigma}^{(1)}|E| \psi_{\sigma}^{(1)}\right\rangle$, is obtained similarly by substituting Eq. (B23), and yields

$$
\left\langle\psi_{\sigma}^{(1)}|E| \psi_{\sigma}^{(1)}\right\rangle=\frac{3}{4} \frac{\epsilon_{5}}{\omega_{5}^{2}}+\frac{1}{\omega_{1}^{2}} \mathcal{E}_{11}-\frac{\Gamma}{\omega_{1}} \mathcal{E}_{10} .
$$

Substituting Eqs. (B26), (B30), and (B31) into Eq. (64) then yields the final result, Eq. (64), listed in the body of the paper. The excess susceptibility (62) is obtained by substituting Eqs. (63) and (64) into (62), and carrying out the $\sigma$ summation with the result

$$
\Delta \chi_{\ell}=\frac{3 \epsilon_{4}}{16 \Gamma \omega_{4}^{2}}+\frac{\mathcal{E}_{11}}{4 \Gamma \omega_{1}^{2}}+\frac{v_{s}^{2} \mathcal{E}_{10}}{2 \Gamma \omega_{1}}\left(\frac{1}{\omega_{1}}-\frac{1}{2}\right) .
$$

The final step is to express the coefficients $\mathcal{E}_{11}$ and $\mathcal{E}_{10}$ in Eq. (B25) in terms of the matrix elements $E_{i j}$ defined in Eqs. (9) and (12). To do so, we write $u_{1}=\left(\frac{1}{2}-v_{s}^{2}\right) u_{0}-\frac{1}{2}\left(1-c^{2}\right)$ and use $\mathcal{E}_{00}=0$ to find

$$
\begin{aligned}
\mathcal{E}_{10} & =-\frac{1}{2}\left\langle 1|E| 1-c^{2}\right\rangle=-\frac{1}{2}\left(E_{00}+6 E_{10}\right), \\
\mathcal{E}_{11} & =\frac{1}{4}\left\langle 1-c^{2}|E| 1-c^{2}\right\rangle-\left(\frac{1}{2}-v_{s}^{2}\right)\left\langle 1|E| 1-c^{2}\right\rangle \\
& =\frac{1}{4} E_{00}-\left(\frac{1}{2}-v_{s}^{2}\right)\left(E_{00}+6 E_{10}\right) .
\end{aligned}
$$


[1] J.R. Dorfman, T.R. Kirkpatrick, and J.V. Sengers, Annu. Rev. Phys. Chem. 45, 213 (1994).

[2] P.L. Garrido, J.L. Lebowitz, C. Maes, and H. Spohn, Phys. Rev. A 42, 1954 (1990).

[3] H. van Beijeren, J. Stat. Phys. 60, 845 (1990).

[4] B. Schmittmann and R.K.P. Zia, in Phase Transitions and Critical Phenomena, edited by C. Domb and J.L. Lebowitz (Academic Press, London, 1995), Vol. 17.

[5] G. Grinstein, D.-H. Lee, and S. Sachdev, Phys. Rev. Lett. 64, 1927 (1990); G. Grinstein, J. Appl. Phys. 69, 5441 (1991).

[6] U. Frisch, D. d'Humières, B. Hasslacher, P. Lallemand, Y. Pomeau, and J.-P. Rivet, Complex Syst. 1, 31 (1987) [reprinted in Lattice Gas Methods for Partial Differential Equations, edited by G. Doolen (Addison-Wesley, Reading, MA, 1990)].

[7] D.H. Rothman and S. Zaleski, Rev. Mod. Phys. 66, 1417 (1994).
[8] H.J. Bussemaker and M.H. Ernst, Physica A 194, 258 (1993); Phys. Lett. A 177, 316 (1993).

[9] H.J. Bussemaker, Phys. Rev. E 53, 1644 (1996).

[10] M.H. Ernst and H.J. Bussemaker, J. Stat Phys. 81, 515 (1995).

[11] H.J. Bussemaker, M.H. Ernst, and J.W. Dufty, J. Stat. Phys. 78, 1521 (1995).

[12] J.R. Dorfman and H. van Beijeren, in Statistical Mechanics, Part B: Time Dependent Processes, edited by B.J. Berne (Plenum Press, New York, 1977).

[13] E.C.G. Stueckelberg, Helv. Phys. Acta 25, 577 (1952).

[14] R. Brito, H.J. Bussemaker, M.H. Ernst, and J. Matsui, Phys. Rev. E 52, 2657 (1995).

[15] R. Brito and M.H. Ernst, J. Phys. A 24, 3331 (1991).

[16] H.J. Bussemaker and M.H. Ernst, Transp. Theory Stat. Phys. 23, 147 (1993).

[17] P. Grosfils, J.P. Boon, R. Brito, and M.H. Ernst, Phys. Rev. E 47, 2473 (1993). 